\title{
The scaling relation of early-type galaxies in clusters
}

\section{Spectroscopic data for galaxies in eight nearby clusters ${ }^{\star} \star \star$}

\author{
D. Bettoni ${ }^{1}$, M. Moles ${ }^{2}$, P. Kjærgaard ${ }^{3}$, G. Fasano ${ }^{1}$, and J. Varela ${ }^{1}$ \\ 1 Osservatorio Astronomico di Padova, Vicolo de'll Osservatorio 5, 35122 Padova, Italy \\ e-mail: [bettoni; fasano; varela]@pd.astro.it \\ 2 Instituto de Astrofísica de Andalucía, Consejo Superior de Investigaciones Científicas, Camino Bajo de Huétor 50, Apdo. 3004 , \\ 18080 Granada, Spain \\ e-mail:moles@iaa.es \\ 3 Copenhagen University Observatory, The Niels Bohr Institute for Astronomy, Physics and Geophysics, Juliane Maries Vej 30 , \\ 2100 Copenhagen, Denmark \\ e-mail: per@astro.ku.dk
}

Received 1 December 2005 / Accepted 22 February 2006

ABSTRACT

\begin{abstract}
Aims. We present low and intermediate resolution spectroscopic data collected for 152 early type galaxies in 8 nearby clusters with $z \leq 0.10$.

Methods. We use low resolution data to produce the redshift and the K-correction for each galaxy, as well as to give their overall spectral energy distribution and some spectral indicators, including the $4000 \AA$ break, the $\mathrm{Mg}_{2}$ strength and the NaD equivalent width. We have also obtained higher resolution data for early type galaxies in three of the clusters, to determine their central velocity dispersion.

Results. The effect of the resolution on the measured parameters is discussed.

Conclusions. A new accurate systemic redshift and velocity dispersion is presented for four of the surveyed clusters, A98, A3125, A3330, and DC2103-39. We have found that the K-correction values for E/S0 bright galaxies in the given nearby clusters are very similar. We also find that the distribution of the line indicators significantly differs from cluster to cluster.
\end{abstract}

Key words. galaxies: elliptical and lenticulars, $\mathrm{cD}$ - galaxies: distances and redshifts - galaxies: clusters: general

\section{Introduction}

This is our second paper presenting data on nearby clusters of galaxies. The motivations and details of the program were given in Fasano et al. (2002, hereafter Paper I; see also Fasano et al. 2000), following the proposal discussed by Kjærgaard et al. (1993, hereafter KJM; see also Moles et al. 1998). They will be used together with the results from the project WINGS (Fasano et al. 2006) to analyze the scaling relations of early-type galaxies in clusters.

Here we present low resolution $(20 \AA$ and $40 \AA$, LRS/20 and LRS/40 respectively in the following) and intermediate resolution spectroscopy ( $3.3 \AA$ resolution, IRS in the following) of 152 early type galaxies in 8 nearby clusters. The LRS data are intended to provide the redshifts of the candidate galaxies, to establish their membership to a given cluster. They also allow the measurement of the K-correction for each individual galaxy and the strength of some spectral features. Rather than the more often used $\mathrm{Mg}_{2}$, we consider here two other prominent spectral features, the NaD doublet and the $4000 \AA$ break, D4000.

* Based on data obtained with the Nordic Optical Telescope (La Palma, Spain) with ALFOSC. Also based on observations obtained with DFOSC at the D1.54m telescope at the European Southern Observatory (La Silla, Chile).

$\star \star$ Tables 3, 4 and 6 are only available in electronic form at http://www. edpsciences.org
The NaD line index is more sensitive to temperature effects than $\mathrm{Mg}_{2}$, but it can be affected by the presence of interstellar material (Burstein et al. 1984; Bica \& Alloin 1986; Bica et al. 1991). However, once allowance is made for that, it correlates well with other indices, $\mathrm{Mg}_{2}$ in particular. Bica \& Alloin (1986) concluded that for a metallicity not greater than solar, any excess of $\mathrm{NaD}$ (with respect to the prediction from the $\mathrm{Mg}_{2}$ indicator) can be due only to interstellar absorption. An outlying position in the $\mathrm{Mg}_{2}-\mathrm{NaD}$ plane can therefore be interpreted as a sign of peculiarity (see Bica et al. 1991).

The $4000 \AA$ break, D4000, is primarily sensitive to the presence of young stars. Hamilton (1985) selected it for that reason to study the evolution of early type galaxies with redshift. Dressler \& Shectman (1987) concluded that it is not sensitive to metallicity but only to the presence of young stars, and insisted on the adequacy of the break indicator to follow the cosmic evolution of early type galaxies. Kimble et al. (1989) found that D4000 does correlate with some metallicity indicators. The theoretical work by Poggianti \& Barbaro (1997) showed that the break is strongly dependent on the effective temperature, and is also sensitive to the metallicity, but only for intermediate temperature stars. On this basis, Barbaro \& Poggianti (1997) developed evolutionary models showing that D4000 would be a measure of the present star formation rate. The calibration of D4000 in terms of the atmospheric stellar parameters is rather complicated as many absorption lines are included in the break. 
Table 1. Log and setup of the observations.

\begin{tabular}{ccccccccc}
\hline \hline Run & Date & Mode & Tel. & "/pix & $\begin{array}{c}\text { Gr. } \\
\#\end{array}$ & $\begin{array}{c}\lambda \\
\text { range }(\AA)\end{array}$ & $\begin{array}{c}\AA / \text { pix } \\
\text { Res } \\
\AA^{a}\end{array}$ \\
\hline 1 & Dec./94 & $L R$ & $D K T$ & 0.49 & 4 & $3300-6400$ & 3.9 & 20 \\
2 & Sep./95 & $L R$ & $D K T$ & 0.49 & 4 & $3300-6400$ & 3.9 & 20 \\
& & $I R$ & $D K T$ & 0.49 & 13 & $4800-5800$ & 0.95 & 3.3 \\
3 & Feb./97 & LR & NOT & 0.188 & 7 & $3800-6800$ & 1.5 & 20 \\
4 & Aug./98 & LR & NOT & 0.188 & 4 & $3300-6400$ & 3.1 & 41 \\
5 & Aug./99 & LR & NOT & 0.188 & 4 & $3300-6400$ & 3.1 & 41 \\
6 & Mar./01 & IR & NOT & 0.188 & 13 & $4800-5800$ & 0.5 & 1.4 \\
\hline
\end{tabular}

${ }^{a}$ Resolution corresponds to the 2". 5 slit used in all the runs.

Gorgas et al. (1999, and references therein) have made an empirical calibration of the break that can be incorporated into the evolutionary models to predict its value. The most interesting aspect of the break indicator is its sensitivity to recent star formation, and therefore its ability to trace evolution.

The intermediate resolution spectroscopy (IRS) data are necessary to determine the central velocity dispersion needed to build the Fundamental Plane. They also provide more accurate spectral line indices within the covered spectral range. Unfortunately the CCDs we used were very noisy at wavelengths shorter than $5200 \AA$. Thus the measurement of the $\mathrm{H} \beta$ and other indices related to rather weak features was uncertain and we do not include them in the present work.

\section{Observations and data reduction}

The observations were carried out during several runs from 1994 with the Danish $1.54 \mathrm{~m}$ telescope (DKT) at La Silla (Chile), and the Nordic Telescope (NOT) at La Palma (Spain). We used identical focal reducer instruments with both telescopes, DFOSC (DKT) and ALFOSC (NOT), equipped with identical grisms. Some of the data were taken using the MOS possibilities with ALFOSC. The different runs and instrumental setups are given in Table 1. In all observations we used a 2'. 5 slit. For the first run the detector was a Thompson $1024 \times 1024 \mathrm{CCD}$ with $19 \mu \mathrm{m}$ pixel, whereas we used various thinned Ford-Loral $2024 \times 2024$ CCDs with $15 \mu \mathrm{m}$ pixel for the other runs.

Template stars, for the measure of radial velocities and the velocity dispersion, of spectral type G8-K3 III, were observed each night as well as nearby galaxies with known and accurate data. These were used as a standard to gauge the accuracy of the calibrations. We also observed several flux standard stars to allow the data to be fully calibrated. Identification and positional information for the cluster galaxies are taken from Dressler (1980). Otherwise we measured the positions on the DSS. In Table 3, in Col. 1 we give the name (from NED or Simbad databases), in Cols. 2 and 3 the coordinates and in Col. 4 the reference identification used for the spectrum, this identification is that used in Tables 4 and 6 to identify the galaxies for clusters A98 and A3330. The identifications in Tables 4 and 6 for galaxies in the remaining clusters are from Dressler (1980).

The data reduction procedure was similar for the LRS and IRS data. It was performed within IRAF ${ }^{1}$. The scientific frames

1 IRAF is the Image Analysis and Reduction Facility made available to the astronomical community by the National Optical Astronomy Observatories, which are operated by the Association of Universities for Research in Astronomy (AURA), Inc., under contract with the US National Science Foundation. were corrected for bias and flat-field and calibrated using a $\mathrm{He} / \mathrm{Ar}$ arc-lamp. For the MOS data we extracted individual 2D spectra for each object and treated these as any other spectra, so we do not refer to them explicitly in the following.

\subsection{The measurement of the redshift. Internal and external accuracy}

Before attempting to measure the redshift of the target galaxies, we checked the accuracy of the zero point in the $\lambda$-calibration of each spectrum, examining the position of the night sky line [OI] $\lambda 5577.32 \AA$. When necessary the spectrum was shifted to that nominal position. For the radial velocity standard stars, given the short exposure time, the night sky lines were not detected so this procedure could not be applied. We decided to place the standards at zero velocity using their own spectral lines; we then used the IRAF cross-correlation package to control the consistency of the method, i.e., that together, they all define a zero velocity system, within the uncertainties.

The standard stars were then used as templates to measure the redshift of the standard galaxies, using the same IRAF packages. The radial velocity was determined as the mean of the results for the different template stars. The typical scatter is $15 \mathrm{~km} \mathrm{~s}^{-1}$. In all cases the scatter was within the formal uncertainties of the parameters. The results for those standard galaxies are given in Table 4.

Comparing the results obtained for the standard galaxies from LRS/20 and IRS data (9 cases; there are no galaxies observed in both IRS and LRS/40 modes) we find that the differences in redshift have an average of $7 \mathrm{~km} \mathrm{~s}^{-1}$, with a scatter of $32 \mathrm{~km} \mathrm{~s}^{-1}$. Part of that scatter is due to one galaxy, E462G15, for which the IRS result is $71 \mathrm{~km} \mathrm{~s}^{-1}$ higher than the LRS one. Notice that the IRS value is much closer to the redshift reported by Jørgensen et al. (1996, JFK) for that galaxy.

To check the external consistency of the data we have compared them with other sources, JFK (10 galaxies in common, 7 with IRS data) and Smith et al. (2000) (7 objects in common, 5 with IRS data). If we compare only our IRS data, the agreement is excellent with both sources, with $\sigma$ (diff.) $\sim 15 \mathrm{~km} \mathrm{~s}^{-1}$. The comparison is also good when LRS data are included, except for EG462G15.

Once the consistency of our measured redshift was assured, we decided to use the standard galaxies as templates to determine the redshift of the target cluster galaxies. We found that they give more accurate results than the standard stars, due to the better spectral matching between both sets of galaxies. Each target object was cross-correlated with all the templates. The average of the resulting $z$ values was taken as the redshift of the galaxy, and the scatter as a quality indicator. The heliocentric redshift values are given in Table 4 . The code 1 to 4 corresponds to $1 \sigma$ values of $\leq 50 \mathrm{~km} \mathrm{~s}^{-1}$, between 50 and $100 \mathrm{~km} \mathrm{~s}^{-1}$, between 100 and $200 \mathrm{~km} \mathrm{~s}^{-1}$, and between 200 and $300 \mathrm{~km} \mathrm{~s}^{-1}$. That scatter is typically smaller than $75 \mathrm{~km} \mathrm{~s}^{-1}$, with more than $75 \%$ of values within $150 \mathrm{~km} \mathrm{~s}^{-1}$, even if it reaches $300 \mathrm{~km} \mathrm{~s}^{-1}$ in some particular cases.

The internal accuracy of the LRS data can be assessed looking at Figs. 1 and 2.

We have searched the literature to examine the external accuracy of our LRS data. The comparison was restricted to those sources for which there are a minimum of 5 galaxies in common. The agreement is in general good as can be seen in Table 2. Our cz values tend to be slightly higher than most of the others. While this cannot be excluded, the differences are about $50 \mathrm{~km} \mathrm{~s}^{-1}$ or 


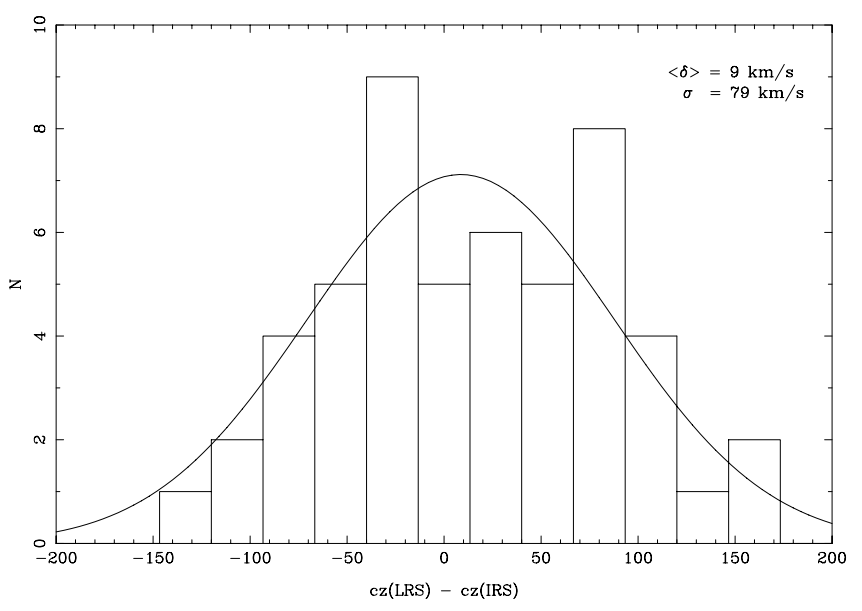

Fig. 1. Distribution of the cz differences for the 52 objects that have both IRS and LRS data. The bin size is $25 \mathrm{~km} \mathrm{~s}^{-1}$. The solid line is the Gaussian fit to the binned data.

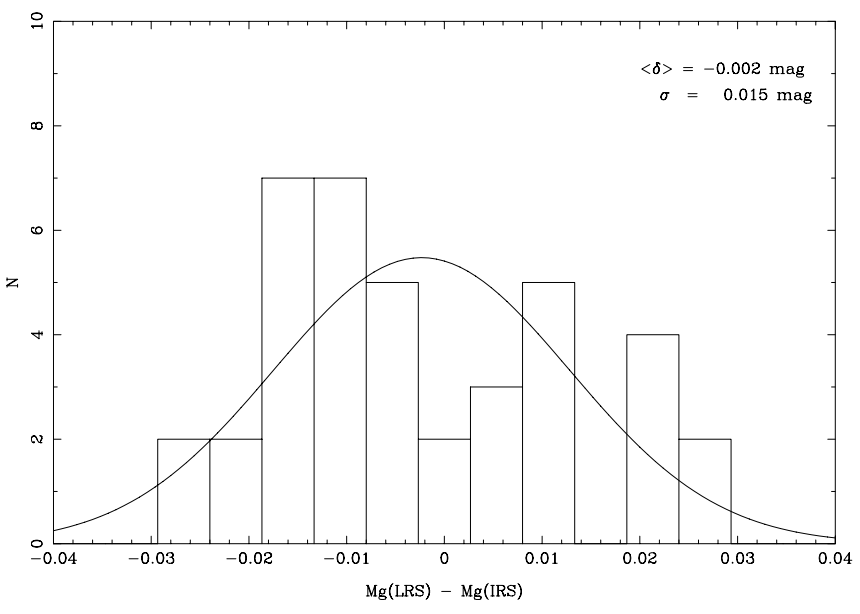

Fig. 2. Distribution of the differences in the $\mathrm{Mg}_{2}$ values for 42 objects for which both IRS and LRS data are available. The bin size is 0.004 . The solid line is the Gaussian fit to the binned data.

less, so we can conclude that our redshift measurements are in the same system as those reported in the quoted references. Indeed, the situation is similar for the IRS data, (see Table 2). The cases for which the disagreement is higher and cannot be accounted for by observational errors have been flagged in Table 4, and commented in the footnotes.

\subsection{The measurement of the central velocity dispersion}

The velocity dispersion of each target galaxy was determined as the mean of the results for the different template stars. The typical scatter is $10 \mathrm{~km} \mathrm{~s}^{-1}$. In all cases the scatter was within the formal uncertainties of the parameters. The velocity dispersion and the radial velocity were derived both for the comparison galaxies and for the target galaxies with the Fourier Quotient Technique (Sargent et al. 1977; Bertola et al. 1984), using the standard stars as templates. The results are presented in Table 4. Comparison with the JFK values shows a good agreement. The differences have an rms value of $15 \mathrm{~km} \mathrm{~s}^{-1}$. Unfortunately the external check can only be done for a handful of target galaxies. We have found independent determinations for only 5 of the galaxies in our sample, all from Wegner et al. (1999). For them, the agreement is also satisfactory since the rms of the differences is the same as for the standard galaxies, $15 \mathrm{~km} \mathrm{~s}^{-1}$.
Table 2. External comparison of the redshift data.

\begin{tabular}{lccccccc}
\hline \hline Cluster & \multicolumn{4}{c}{ LRS } & \multicolumn{4}{c}{ IRS } \\
& Ref. & $N$ & $\Delta(c z)$ & $\sigma$ & $N$ & $\Delta(c z)$ & $\sigma$ \\
\hline$A 98$ & 1 & 10 & +36 & 147 & - & - & - \\
& 2 & 11 & -10 & 120 & - & - & - \\
$A 119$ & 3 & 14 & +52 & 75 & 11 & 80 & 48 \\
& 4 & 6 & +32 & 91 & 6 & 46 & 57 \\
& 5 & 5 & +3 & 56 & 5 & 28 & 52 \\
$A 3125$ & 8 & 6 & -11 & 121 & 5 & -17 & 133 \\
$A 1069$ & 3 & 7 & +29 & 116 & - & - & - \\
& 6 & 6 & +15 & 162 & - & - & - \\
$A 1983$ & 7 & 14 & +31 & 77 & - & - & - \\
$A 2151$ & 7 & 9 & -15 & 153 & - & - & - \\
\hline
\end{tabular}

References. 1, Beers et al. (1982); 2, Zabludoff et al. (1990); 3, Katgert et al. (1998); 4, Wegner et al. (1999); 5, Huchra et al. (1999); 6, Beers et al. (1991); 7, Dressler \& Shectman (1988); 8, Caldwell \& Rose (1997).

\subsection{The measurement of the K-effect and of the spectral features}

To measure the spectral characteristics, the spectra were first shifted to zero redshift, using the measured $c z$ values with the appropriate tasks within IRAF. Then, the K-effect was measured by computing the magnitude in a given band in both the observed and the zero-redshifted spectra. Given the spectral coverage of our data and the redshift of the sources, it was not always possible to compute the K-effect for the three $B, V$ and Gunn $r$ bands. The only band for which we have measured the K-effect for all the targets is $V$. The results are given in Table 4 .

In Table 5 we present the average values for each cluster we have observed. Comparing with the values reported by Pence (1976) for E/S0 galaxies, and with the model predictions by Poggianti (1997) for the same type of galaxies, we find a very good agreement. We notice that the scatter of the K-corrections for early type galaxies in clusters is very small, indicating that applying the same correction to all the bright E/S0 galaxies in clusters introduces only small errors.

To measure the $4000 \AA$ break we chose to use bands of $100 \AA$ width on both sides of the feature. This choice is practical, due to the fact that our spectra do not extend enough into the blue. The data are also collected in Table 4. Even if we cannot directly compare with similar measurements from other authors, we have verified that the range of our values is similar to that found by Dressler \& Shectman (1988) for similar galaxies in nearby clusters. For the different line indicators we have used the Lick spectral bands as defined in Worthy et al. (1994). For reasons we have already indicated, only the most prominent features, $\mathrm{Mg}_{2}, \mathrm{NaD}$, and the $4000 \AA$ break were measured. The results are presented in Table 4.

Considering the $\mathrm{Mg}_{2}$ strength measurements the internal accuracy can be ascertained from repeated measurements with the same resolution. The rms of the differences for galaxies observed twice in the LRS/20 mode is 0.014 , most probably due to the poor $\mathrm{S} / \mathrm{N}$ of some of the spectra we repeated. Thus, as for the redshift, we consider that the overall quality of the data is definitely better than that figure. For the standard galaxies we find a good agreement with JFK values, even if the resolution used is not the same. We notice that the same aperture was used by JFK and here. Except for N1403, for which the $\mathrm{Mg}_{2}$ value was quoted as uncertain by JFK, as is our own value, due to a rather 
Table 5. Average values for the clusters.

\begin{tabular}{lcccccccc}
\hline \hline Cluster & $c z$ & $\sigma$ & $\mathrm{Mg}_{2}$ & $\mathrm{NaD}$ & $\mathrm{D} 4000$ & $k_{B}$ & $k_{V}$ & $k_{r}$ \\
\hline A98 & 31225 & 895 & $0.182-0.346(0.280)$ & $3.1-6.4(4.0)$ & $1.45-2.34(1.95)$ & $0.52(0.06)$ & - & - \\
$\mathrm{A} 119$ & 13260 & 773 & $0.195-0.348(0.303)$ & $2.7-5.3(4.1)$ & $1.03-2.06(1.84)$ & $0.22(0.03)$ & $0.07(0.02)$ & $0.03(0.01)$ \\
$\mathrm{A} 3125$ & 17898 & 779 & $0.208-0.358(0.294)$ & $2.3-5.0(3.5)$ & $1.12-2.39(1.64)$ & - & $0.13(0.01)$ & $0.09(0.01)$ \\
A3330 & 27000 & 695 & $0.267-0.365(0.299)$ & $2.1-6.0(4.0)$ & $1.09-2.75(1.63)$ & - & $0.14(0.03)$ & - \\
A1069 & 19671 & 659 & $0.175-0.354(0.269)$ & $2.0-5.5(3.9)$ & $1.06-2.50(1.44)$ & - & $0.10(0.01)$ & $0.07(0.01)$ \\
A1983 & 13080 & 948 & $0.182-0.334(0.309)$ & $2.5-5.5(4.5)$ & $1.49-2.39(1.99)$ & - & $0.07(0.01)$ & - \\
A2151 & 10980 & 716 & $0.264-0.415(0.320)$ & $2.7-6.0(4.1)$ & $1.91-2.44(2.06)$ & $0.19(0.01)$ & $0.06(0.01)$ & - \\
DC2103 & 15268 & 449 & $0.220-0.338(0.282)$ & $1.3-4.5(3.7)$ & $1.11-1.70(1.37)$ & $0.22(0.02)$ & $0.09(0.02)$ & $0.04(0.01)$ \\
\hline
\end{tabular}

The values of $c z$ and $\sigma$ for A98, A3125, A3330, and DC2103 are from the present work. For the other clusters they have been taken from Struble $\& \operatorname{Rood}(1999)$.

For the spectral line indicators we give the range and the median value (in parenthesis). For the K-correction only the median value is given.

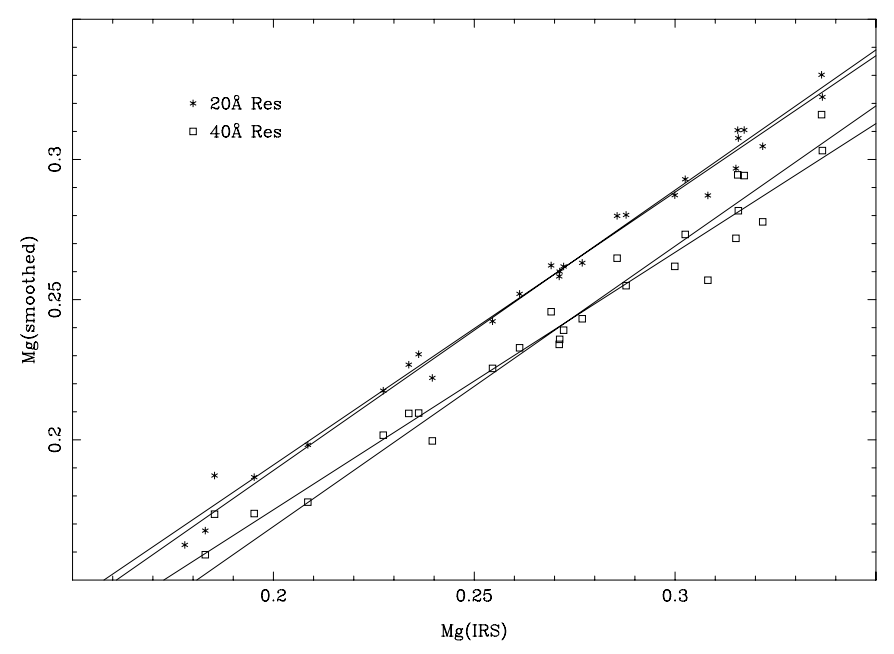

Fig. 3. The relation between the $\mathrm{Mg}_{2}$ strength values obtained from different resolution data. The plotted lines for the two sets of data are the best fit and the fit with slope $=1$ in each case.

poor $\mathrm{S} / \mathrm{N}$, the rms value of the differences is slightly smaller than $0.01 \mathrm{mag}$, i.e., smaller than for repeated measurements. As we will discuss below, this difference mainly reflects the effect of the resolution. We notice that the comparison shows that our results are in the same system as those reported by JFK.

The effect of the resolution on the measured values can be checked comparing the results from LRS/40, LRS/20 and IRS data. Starting with the standard galaxies, for the 8 objects measured in LRS/20 and IRS modes, the average difference is $0.004 \mathrm{mag}$, with an rms value of $0.014 \mathrm{mag}$. Much of that scatter is due to a single object, N1395, for which the LRS value is $0.028 \mathrm{mag}$ smaller than the IRS value. Regarding the target galaxies, we have 42 objects for which we could measure the $\mathrm{Mg} 2$ strength from LRS and IRS data. Excluding three grossly discrepant cases (more than 0.03 mag difference), the differences have an average of $0.003 \mathrm{mag}$, with $\sigma=0.014 \mathrm{mag}$. Their median value is 0.006 mag (see Fig. 2).

To further test the effect, we have degraded the observed IRS data to produce spectra with the resolution of the LRS/20 and LRS/40 modes. The results are presented in Fig. 3. The relations have slopes very close to 1 , in particular between LRS/20 and IRS data. The systematic effect can be represented by the relation $\mathrm{Mg}_{2}(\mathrm{IRS})=\mathrm{Mg}_{2}(\mathrm{LRS} / 20)+0.011 \mathrm{mag}$, with a scatter of $0.004 \mathrm{mag}$. For the LRS $/ 40$ data the correction is $0.031 \mathrm{mag}$, with a scatter of $0.007 \mathrm{mag}$.

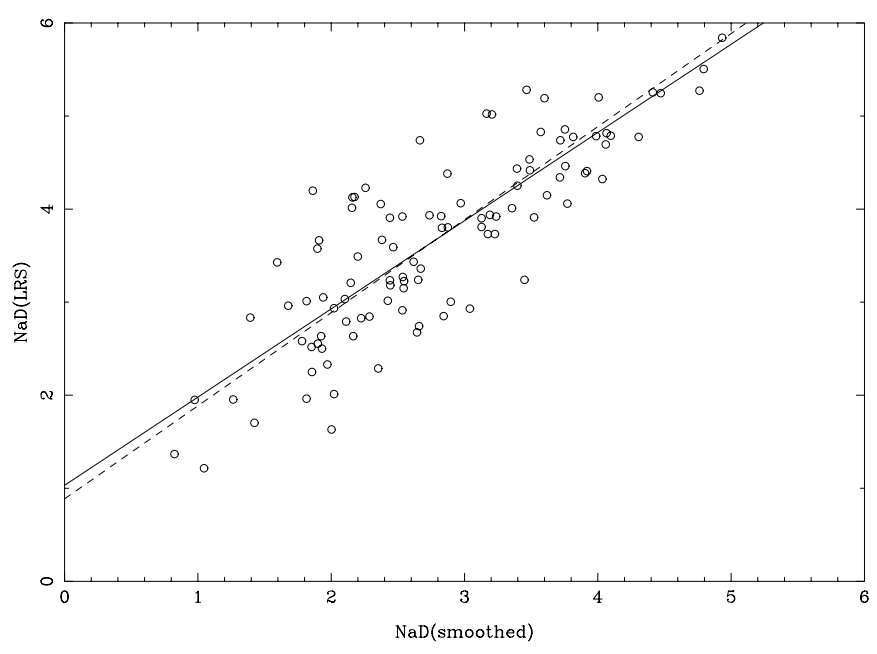

Fig. 4. The EW of the NaD line from real LRS/20 data and from the same spectra degraded to $40 \AA$ resolution. The lines are the best fit and the fit with slope $=1$.

For the other line indicators, we estimate that the errors are of the order of $5 \%$ at worst, a figure that is confirmed by the comparison of the values obtained from repeated measurements. The $4000 \AA$ break is a very robust indicator that is not affected by other aspects, whereas an important resolution effect is expected on the $\mathrm{NaD}$ values since the Lick bands used to define the index are rather narrow. Given that the wavelength range of the IRS data does not cover the $\mathrm{NaD}$ region, and we do not have enough data taken in both LRS modes for a sound comparison we have simply measured the $\mathrm{NaD}$ equivalent width for the LRS/20 data degraded to the resolution of the runs 4 and 5, namely $40 \AA$. The results are plotted in Fig. 4. We find that the correction is of the form $E W(\mathrm{LRS} / 20)=E W(\mathrm{LRS} / 40)+0.9 \AA$. This is the correction we will apply to put all the $\mathrm{NaD} E W$ values in the same LRS/20 system.

\subsection{Aperture corrections: the final data}

To perform the aperture correction of the $\mathrm{Mg}_{2}$ index we have followed JFK to transform the observed values to the standard aperture of $1.19 h^{-1} \mathrm{kpc}$, corresponding to $3 . \prime 4$ at the distance of Coma. Their expression (4) was used to evaluate the correction. As we have already noticed, our data are in a system similar to that of JFK, so we have still to transform our values to put them on the Lick system. This has been done also following JFK, 
adding 0.011 to our values. Finally, we have not corrected for the velocity dispersion simply because it is not known for most of our galaxies; for a correction such as that proposed by JFK, it would never be greater than 0.003 .

Similarly, aperture corrections should be applied to the other line indicators. For the $\mathrm{NaD}$ equivalent width, given that there are no specific data on its radial gradient, we decided to apply the same kind of correction as for the $\mathrm{Mg}_{2}$ index. The final expression for the aperture corrected $\mathrm{NaD}$ line $E W$ is given by

$(E W)_{n}=W\left[1-\left(r_{\mathrm{o}} / r_{n}\right)^{-0.016}\right]+(E W)_{\mathrm{o}} \times\left(r_{\mathrm{o}} / r_{n}\right)^{-0.016}$

where the index $n(\mathrm{o})$ stands for aperture corrected (observed) value, and $W$ is the width of the filter used to measure the feature $(32.5 \AA$ in the system defined by Worthy et al. 1994). The maximum correction, for $\mathrm{A} 98$, is $0.60 \AA$, whereas it is of $0.18 \AA$ for A119.

For the aperture correction of the $4000 \AA$ break we have used the result by Sánchez-Blázquez et al. (2001), who have found that the break changes as $-0.20 \log (r)$. The maximum correction is 0.11 .

The final, corrected values for the redshift, the velocity dispersion and the line indicators are given in Table 6. We have taken the $\mathrm{Mg}_{2}$ results from the IRS mode when available, correcting the values obtained with LRS data to that resolution following the methods discussed before, including the correction to place them in the Lick system. For the $\mathrm{NaD} E W$ value, we have corrected all the LRS/40 measurements to LRS/20 by adding $0.90 \AA$ to take into account the resolution effect as discussed before.

\section{General considerations}

\subsection{The redshift of the clusters}

For four clusters, namely A119, A1069, A1983 and A2151, the number of new (or significantly modified) redshift values we can add represents only a small fraction of the total known. There are some cases however, namely A98, A3125, A3330, and DC2103 where that number is significantly increased. Therefore, a new and more precise determination of their redshift and velocity dispersion is possible. We discuss them briefly below. The results are given in Table 5 .

We have obtained the redshift for 31 galaxies in the field of A98 (see Table 5), of which 16 are also in Beers et al. (1982), or in Zabludoff et al. (1990), or in both. Following the preceding discussion on the redshift accuracy of our data, we have adopted our values in all cases when they were also given in other references. The redshift distribution of all the galaxies with known $\mathrm{cz}$ in the field of A98 is presented in Fig. 5. Considering the 36 objects with cz in the range between $29500 \mathrm{~km} \mathrm{~s}^{-1}$ and $33000 \mathrm{~km} \mathrm{~s}^{-1}$, we find for the cluster redshift $c z=31225 \mathrm{~km} \mathrm{~s}^{-1}$, with $\sigma=895 \mathrm{~km} \mathrm{~s}^{-1}$. We notice that 6 of the 7 foreground galaxies define a group with $c z=17973 \mathrm{~km} \mathrm{~s}^{-1}$, and $\sigma=254 \mathrm{~km} \mathrm{~s}^{-1}$.

Beers et al. (1982) argued that A98 would comprise two spatially and dynamically distinct extensions. With the new data, a total of 11 galaxies are located in the A98N extension, and 25 in A98S. The velocity distribution shown in Fig. 5 shows only one peak and is rather smooth. There is a slight redshift difference between the northern and southern extensions, but is not significant. Therefore, the existence of two substructures in $c z$ space is not confirmed with the still scarce data available.

Considering A3125, Caldwell \& Rose (1997) have reported the redshift of 16 galaxies in the field of the cluster. Only five are
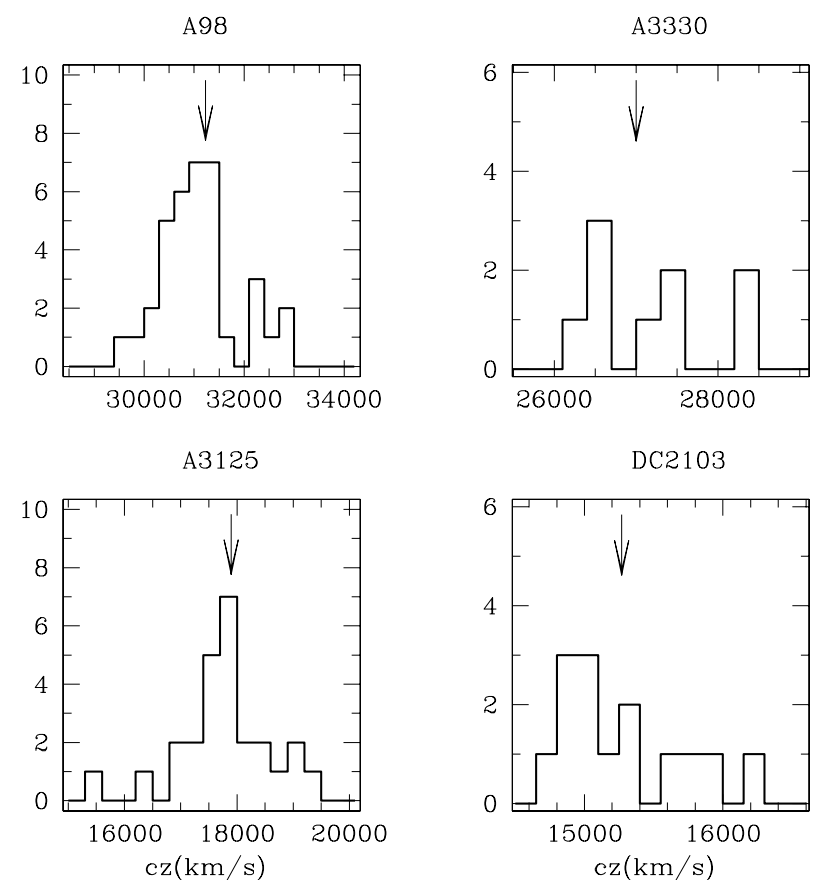

Fig. 5. Distribution of known redshift of galaxies in the clusters A3125, DC2103, A3330 and A98. The arrows indicate the cluster mean cz value as given in the text.

also in our sample. Together with our measurements, the total amounts now to 31 values. The $c z$ distribution is given in Fig. 5. Taking out the 5 outliers ( 2 fore- and 3 background galaxies), we are left with 26 objects, which define the cluster redshift $c z=$ $17898 \mathrm{~km} \mathrm{~s}^{-1}$, with $\sigma=779 \mathrm{~km} \mathrm{~s}^{-1}$.

The redshift quoted for A3330 in the compilation by Struble $\& \operatorname{Rood}(1999), z=0.0921$, was determined with only two galaxies (Ebeling \& Maddox 1997). From the 9 galaxies we have measured, we find $c z=27000 \mathrm{~km} \mathrm{~s}^{-1}$, and $\sigma=695 \mathrm{~km} \mathrm{~s}^{-1}$, with the distribution plotted in Fig. 5.

The situation is similar for the cluster DC2103-39, for which only 3 redshifts were known (Loveday et al. 1996). We have measured the redshift of 21 galaxies in the area, of which 6 are foreground and 1 is a background object. For the remaining 14 objects (see Fig. 5) we find $c z=15268 \mathrm{~km} \mathrm{~s}^{-1}$, with $\sigma=449 \mathrm{~km} \mathrm{~s}^{-1}$. All the six foreground objects are at a similar redshift. Five of them are grouped with an average $c z=$ $9260 \mathrm{~km} \mathrm{~s}^{-1}$, with $\sigma=70 \mathrm{~km} \mathrm{~s}^{-1}$. These galaxies are located in the field we called " $a$ " in Paper I. They could be part of a cluster as more galaxies are visible in that field.

\subsection{The range of spectral properties}

In Table 5 we also give the range and median values of the $\mathrm{K}$-correction terms and the spectral indicators we have considered here. The K-correction values, as indicated before, are very similar for all the bright $\mathrm{E}$ and $\mathrm{S} 0$ galaxies in a given cluster. The average values are very well defined, with a small scatter, and follow the trend with $z$ shown by the data presented by Pence (1976) and with the model prediction by Poggianti (1997) for the same kind of galaxies.

The $\mathrm{Mg}_{2}$ values span a rather large range in all the 8 clusters (see Fig. 6), in most cases from 0.20 to 0.35 . The exceptions are A2151 and A3330 with higher minimum values, what could be due to the small number of objects observed in these clusters. The median values are however different, from 


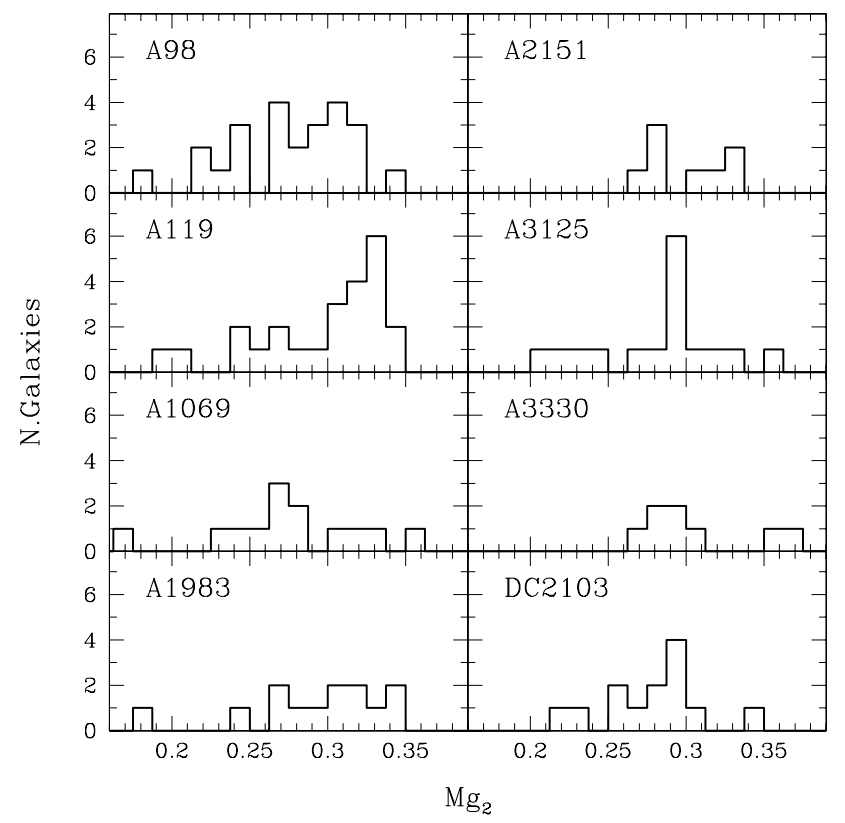

Fig. 6. Distribution of the $\mathrm{Mg}_{2}$ strength for all the measured galaxies in each cluster.

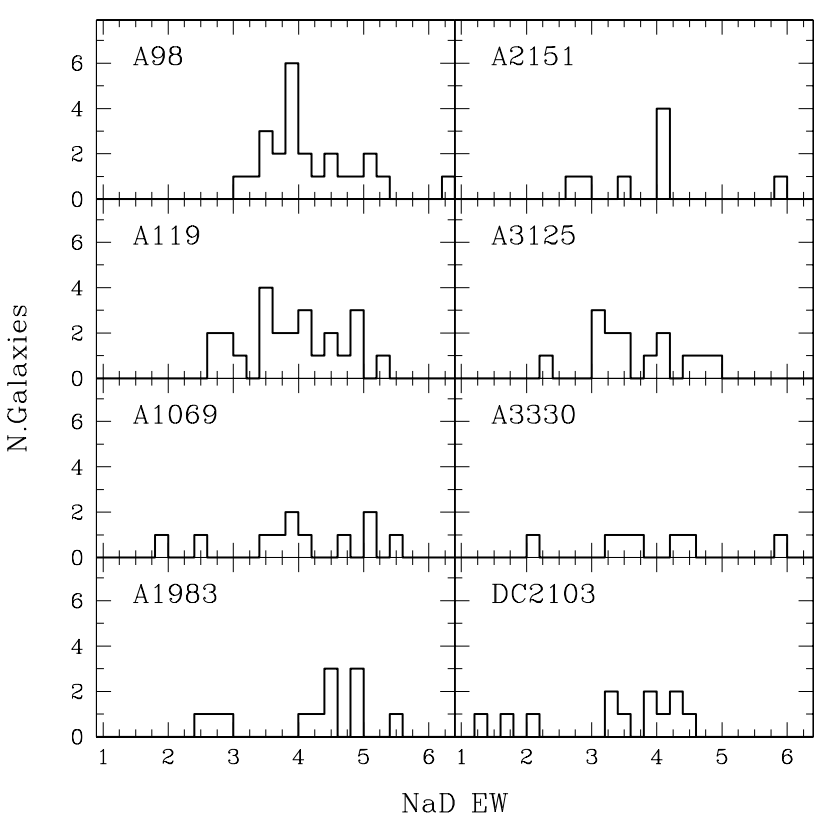

Fig. 7. Distribution of the $\mathrm{NaD}$ equivalent width for all the measured galaxies in each cluster.

0.269 mag in A1069 to 0.320 mag in A2151. There is no significant trend with $c z$, finding clusters at similar $c z$ with different median values. The situation is similar for the $\mathrm{NaD}$ (Fig. 7) equivalent width, which also spans a large range of values in each cluster. The median values are however within $1 \AA$. Finally, for the $4000 \AA$ break (Fig. 8), there are differences in the ranges, but could be simply due to the small number of sources. We point out the absence of low D4000 values in A2151, and that of high values in DC2103. We notice that a trend is visible between the $\mathrm{NaD}$ and $\mathrm{Mg}_{2}$ indicator, but with an important scatter (see Fig. 9).

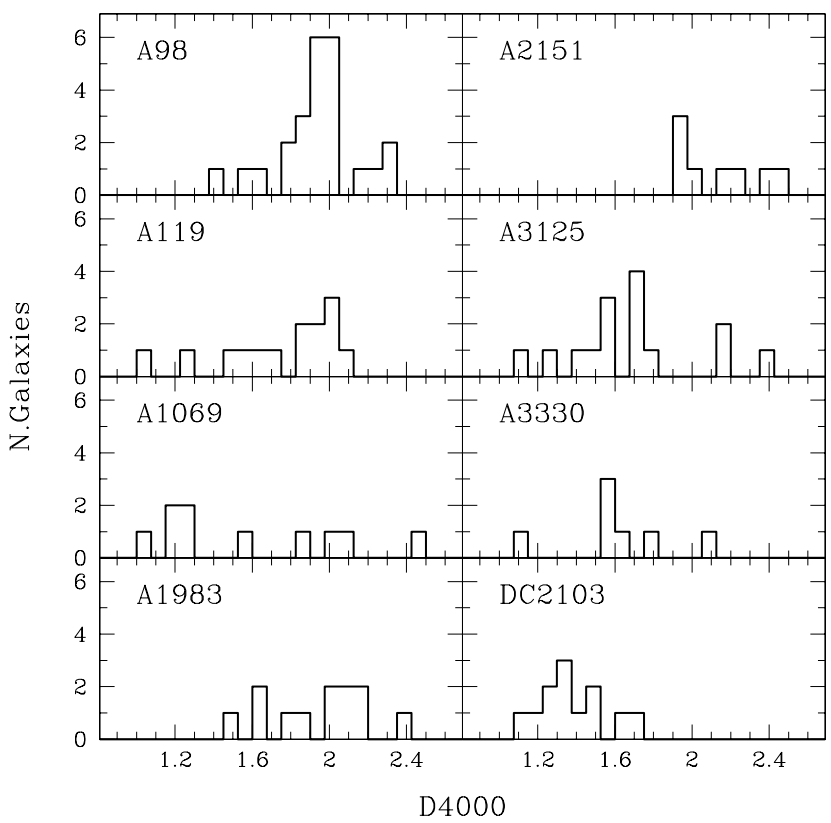

Fig. 8. Distribution of the the $4000 \AA$ break line indicator for all the measured galaxies in each cluster.

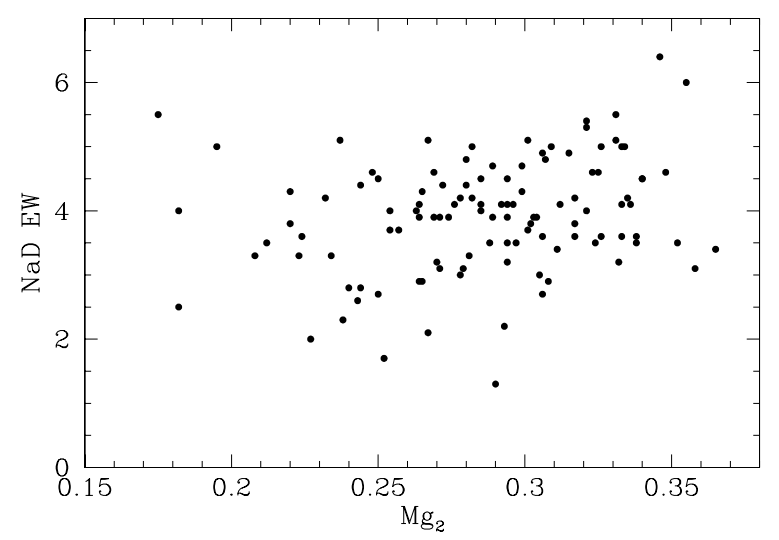

Fig. 9. The $\mathrm{Mg}_{2}$ strength versus the $\mathrm{NaD}$ equivalent length for the galaxies in different clusters.

\subsection{Galaxies with emission lines}

The targets we selected were among the brightest cluster galaxies classified as E or S0, so we did not expect to find many showing emission lines. Out of a total of 13 of such objects objects, half are actually foreground objects relative to the cluster under consideration. These are A1069d41, A3125d11, A3125d14, A98F3g4, and DC2103D18. They all have typical HII-like spectra.

The cluster member galaxies with emission lines are the following:

- A119d44. Faint [OIII] 25007 detected only in the IRS data.

- A119d45. This galaxy presents a spectrum typical of a star forming region.

- A3125d160.Faint [OIII] $\lambda 5007$ detected only in the IRS data.

- A3125d77. It presents strong $\mathrm{H} \alpha$ emission, with some structure. There is some indication of high electronic density from the [SII] lines. Neither the [OIII] lines, nor $\mathrm{H} \beta$ are visible in our spectrum.

- A2151d78. This galaxy (IC 1182) is a known peculiar early type object, with a spectrum in the border line between star 
forming and active galaxies. Its properties have been discussed in Moles et al. (2004).

- A98F1g44. It presents all the lines typical of star forming galaxies, from [OII] to [SII].

- A98F1g58. Spectrum typical of an early type galaxy, but with $\mathrm{H} \alpha$, [NII] and [SII] clearly visible.

- DC2103d18. Faint [OIII] $\lambda 5007$ detected only in the IRS data.

We notice that all have spectra of star forming regions, and none shows signs of nuclear activity.

\section{Summary and conclusions}

We present here LRS and/or IRS for 152 early type galaxies in 8 nearby clusters with $z \leq 0.1$. Data on the redshift, velocity dispersion (for a sub sample), the K-correction, and the most prominent spectral indicators are given.

Comparison of LRS results with IRS and literature data indicate that low resolution spectra can provide accurate values for the redshift (within $65 \mathrm{~km} \mathrm{~s}^{-1}$ ), and for the $\mathrm{Mg}_{2}$ strength (within $0.01 \mathrm{mag}$ ). Moreover, since the high and moderate resolution measurements are tightly related, a well defined correction can be applied to obtain all the data in a common base. This means that accurate enough data can be obtained with moderate resolutions, and therefore for galaxies to rather high redshift, an important point for any evolutionary study.

Our data have allowed us to give more precise values for the redshift and velocity dispersion of 4 clusters, poorly known to date, namely A98, A3125, A3330, and DC2103 (see Table 5).

Regarding the K-correction terms, our results indicate that it is very similar for all the bright $\mathrm{E} / \mathrm{S} 0$ galaxies in a given cluster, so the same correction can be applied to all for many studies. Our values are in very good agreement with the data by Pence (1977) and with the model predictions by Poggianti (1997).

Given the short range in redshift of the clusters analyzed here, no trend with $z$ was expected for any of the spectral indicators, as it is found. It is interesting to note, however, that for all three spectral indicators we find a large range of values for each cluster. It is that physical variance in the spectral properties of the member galaxies that seems to be the most interesting aspect for the present analysis. A trend between the line indicators $\mathrm{NaD}$ and $\mathrm{Mg}_{2}$ is present in our data, in agreement with previous results. The $4000 \AA$ break does not seem to have a clear relation with the other indicators.

\section{References}

Barbaro, G., \& Poggianti, B. 1997, A\&A, 324, 490

Beers, T. C., Geller, M. J., \& Huchra, J. P. 1982, ApJ, 257, 23
Beers, T. C., Gebhardt, K., Forman, W., Huchra, J. P., \& Jones, C. 1991, AJ, 102, 1581

Bertola, F., Bettoni, D., Rusconi, L., \& Sedmak, G. 1984, AJ, 89, 356

Bica, E. L. D., \& Alloin, D. 1986, A\&A, 166, 83

Bica, E. L. D., Pastoriza, M., Maia, M., da Silva, L., \& Dottori, H. 1991, AJ, 102,1072

Bureau, M., Mould, J. R., \& Staveley-Smith, L. 1996, ApJ, 463, 60

Burstein, D., Faber, S. M., Gaskell, C. M., \& Krumm, N. 1984, ApJ, 287, 586

Caldwell, N., \& Rose, J. A. 1997, AJ, 113, 492

da Costa, L. N., Pellegrini, P. S., Davis, M., et al. 1991, ApJS, 75, 935

Davoust, E., \& Considere, S. 1995, A\&AS, 110, 19D

van Dokkum, P. G., \& Franx, M. 1996, MNRAS, 281, 985

Dressler, A. 1980, ApJS, 42, 565

Dressler, A., \& Shectman, S. A. 1987, AJ, 94, 899

Dressler, A., \& Shectman, S. A. 1988, AJ, 95, 284

Ebeling, H., \& Maddox, S. J. 1995, MNRAS, 275, 1155

Falco, E. E., Kurtz, M. J., Geller, M. J., et al. 1999, PASP, 111, 438

Fasano, G., Poggianti, B. M., Couch, W. J., et al. 2000, ApJ, 542, 673

Fasano, G., Bettoni, D., D’Onofrio, M., Kjærgaard, P., \& Moles, M. 2002, A\&A, 387, 26 (Paper I)

Fasano, G., Marmo, C., Varela, J., et al. 2006, A\&A, 445, 805

Fouqué, P., Gourgoulhon, E., Chamaraux, P., \& Paturel, G. 1992, A\&AS, 93, 211

Gorgas, J., Cardiel, N., Pedraz, S., \& González, J. J. 1999, A\&AS, 139, 29

Graham, A. W., Colless, M. M., Busarello, G., Zaggia, S., \& Longo, G. 1998, A\&AS, 133, 325

Hamilton, D. 1985, ApJ, 297, 371

Huchra, J. P., Vogeley, M. S., \& Geller, M. J. 1999, ApJS, 121, 287

Jørgensen, I., Franx, M., \& Kjærgaard, P. 1996, MNRAS, 280, 167 (JFK)

Katgert, P., Mazure, A., Perea, J., et al. 1996, A\&A, 310, 8

Katgert, P., Mazure, A., den Hartog, R., et al. 1998, A\&AS, 129, 399

Kimble, R. A., Davidsen, A. F., \& Sandage, A. R. 1989, ApJS, 157, 237

Kjærgaard, P., Jørgensen, I., \& Moles, M. 1993, ApJ, 418, 617 (KJM)

Lauberts, A., \& Valentjin, E. A. 1989, The Surface Photometry Catalogue of the ESO-Uppsala Galaxies, Garching bei München, ESO

Loveday, J., Efstathiou, G., Maddox, S. J., \& Peterson, B. A. 1996, ApJ, 468, 1

Moles, M., Campos, A., Kjaegaard, P., Fasano, G., \& Bettoni, D. 1998, ApJ, 495, L31

Moles, M., Bettoni, D., Fasano, G., Kjaegaard, P., Varela, J., \& Milvang-Jensen, B. 2004, A\&A, 418, 495

Paturel, G., Petit, C., Prugniel, P., et al. 2003, A\&A, 412, 45

Pence, W. 1976, ApJ, 203, 39

Poggianti, B. M., \& Barbaro, G. 1997, A\&A, 325, 1025

Poggianti, B. M. 1997, A\&AS, 122, 399

Postman, M., \& Lauer, T. R. 1995, ApJ, 440, 28

Sánchez-Blázquez, P., Gorgas, J., Cardiel, N., et al. 2001, Ap\&SS, 277, 351

Sargent, W. L. W., Schechter, P. L., Boksenberg, A., \& Shortridge, K. 1977, ApJ, 212,326

Simien, F., \& Prugniel, P. 1997, A\&AS, 126, 519

Smith, R. J., Lucey, J. R., Hudson, M. J., Schlegel, D. J., \& Davies, R. L. 2000, MNRAS, 313, 469

Struble, M. F., \& Rood, H. J. 1999, ApJS, 125, 35

de Vaucouleurs, G., de Vaucouleurs, A., Corwin, Jr., H. G., et al. 1991, Third Reference Catalogue of Bright Galaxies, RC3

Wegner, G., Colless, M., Saglia, R. P., et al. 1999, MNRAS, 305, 259

Worthy, G., Faber, S. M., González, J., \& Burstein, D. 1994, ApJS, 94, 687

Zabludoff, A. I., Huchra, J. P., \& Geller, M. J. 1990, ApJS, 74, 1

Zabludoff, A. I., Geller, M. J., Huchra, J. P., Vogeley, M. S. 1993, AJ, 106, 1273 
D. Bettoni et al.: Spectroscopic data for galaxies in eight nearby clusters, Online Material $p 1$

\section{Online Material}


D. Bettoni et al.: Spectroscopic data for galaxies in eight nearby clusters, Online Material p 2

Table 3. Galaxies not included in Dressler (1980).

\begin{tabular}{|c|c|c|c|}
\hline Galaxy & $\alpha(2000)$ & $\delta(2000)$ & Spectrum id. \\
\hline \multicolumn{4}{|l|}{ Abell 98} \\
\hline $2 M A S X J 00463567+2029428$ & $00: 46: 35.7$ & $+20: 29: 42$ & $F 1 g 20$ \\
\hline $2 M A S X J 00463524+2030108$ & $00: 46: 35.3$ & $+20: 30: 11$ & $F 1 g 22$ \\
\hline$A 98:[B G H 82] 320$ & $00: 46: 24.7$ & $+20: 30: 07$ & $F 1 g 30$ \\
\hline $2 M A S X J 00462380+2030006$ & $00: 46: 23.9$ & $+20: 30: 00$ & $F 1 g 31$ \\
\hline $2 M A S X J 00462059+2029076$ & $00: 46: 20.7$ & $+20: 29: 07$ & $F 1 g 38$ \\
\hline $2 M A S X J 00461931+2029416$ & $00: 46: 19.4$ & $+20: 29: 41$ & $F 1 g 43$ \\
\hline$A 98:[B G H 82] 311$ & $00: 46: 16.0$ & $+20: 30: 19$ & $F 1 g 44$ \\
\hline $2 M A S X J 00463702+2026068$ & $00: 46: 37.1$ & $+20: 26: 06$ & $F 1 g 58$ \\
\hline $2 M A S X J 00462586+2027326$ & $00: 46: 25.9$ & $+20: 27: 32$ & $F 1 g 65$ \\
\hline $3 C 021$ & $00: 46: 29.4$ & $+20: 28: 04$ & $F 1 g 76$ \\
\hline $2 M A S X J 00463318+2027518$ & $00: 46: 33.2$ & $+20: 27: 51$ & $F 1 q 77$ \\
\hline $2 M A S X J 00463183+2028118$ & $00: 46: 31.9$ & $+20: 28: 11$ & $F 1 g 80$ \\
\hline $2 M A S X J 00463624+2028268$ & $00: 46: 36.3$ & $+20: 28: 26$ & $F 1 g 88$ \\
\hline $2 M A S X J 00463624+2028268$ & $00: 46: 08.5$ & $+20: 28: 50$ & $F 2 g 01$ \\
\hline $2 M A S X J 00460743+2028486$ & $00: 46: 07.5$ & $+20: 28: 48$ & $F 2 g 02$ \\
\hline$A 98:[P B L 2000] 0264$ & $00: 46: 04.8$ & $+20: 28: 27$ & $F 2 g 03$ \\
\hline $2 M A S X J 00455043+2027487$ & $00: 45: 50.5$ & $+20: 27: 49$ & $F 2 g 05$ \\
\hline $2 M A S X J 00455043+2027487$ & $00: 45: 50.4$ & $+20: 29: 08$ & $F 2 g 07 A$ \\
\hline $2 M A S X J 00455008+2029097$ & $00: 45: 50.2$ & $+20: 29: 09$ & $F 2 g 07 B$ \\
\hline $2 M A S X J 00460209+2030516$ & $00: 46: 02.2$ & $+20: 30: 50$ & $F 2 g 09$ \\
\hline $2 M A S X J 00461355+2034516$ & $00: 46: 13.6$ & $+20: 34: 51$ & $F 3 g 01$ \\
\hline $2 M A S X J 00460400+2034516$ & $00: 46: 04.0$ & $+20: 34: 51$ & $F 3 g 02$ \\
\hline$A 98:[B G H 82] 182$ & $00: 45: 59.8$ & $+20: 35: 09$ & $F 3 g 03$ \\
\hline$N P M 1 G+20.0024$ & $00: 45: 52.9$ & $+20: 35: 13$ & $F 3 g 04$ \\
\hline$A 98:[P B L 2000] 376$ & $00: 45: 57.5$ & $+20: 36: 56$ & $F 3 q 05$ \\
\hline $2 M A S X J 00460400+2034516$ & $00: 46: 04.5$ & $+20: 36: 45$ & $F 3 g 07$ \\
\hline$A 98:[P B L 2000] 269$ & $00: 46: 17.1$ & $+20: 23: 44$ & $F 10 g 5$ \\
\hline $2 M A S X J 00461469+2023426$ & $00: 46: 14.7$ & $+20: 23: 42$ & $F 10 g 6$ \\
\hline $2 M A S X J 00462849+2023488$ & $00: 46: 28.5$ & $+20: 23: 48$ & $F 10 g 7$ \\
\hline $2 M A S X J 00463390+2023548$ & $00: 46: 34.0$ & $+20: 23: 55$ & $F 10 g 8$ \\
\hline $2 M A S X J 00463852+2022538$ & $00: 46: 38.6$ & $+20: 22: 53$ & $F 10 g 9$ \\
\hline \multicolumn{4}{|l|}{ Abell 3330} \\
\hline $2 M A S X J 05145263-4859071$ & $05: 14: 52.5$ & $-48: 59: 07$ & $g 21$ \\
\hline $2 M A S X J 05145588-4859121$ & $05: 14: 55.7$ & $-48: 59: 12$ & 922 \\
\hline $2 M A S X J 05150675-4901321$ & $05: 15: 06.6$ & $-49: 01: 33$ & $g 5$ \\
\hline $2 M A S X J 05150726-4902261$ & $05: 15: 07.2$ & $-49: 02: 27$ & $g 6$ \\
\hline$A P M U K S(B J) B 051342.65-490606.2$ & $05: 15: 00.7$ & $-49: 02: 48$ & 97 \\
\hline FAIRALL0790 & $05: 14: 39.4$ & $-49: 03: 29$ & $g 1$ \\
\hline $2 M A S X J 05143494-4905153$ & $05: 14: 34.9$ & $-49: 05: 15$ & $g 2$ \\
\hline $2 M A S X J 05150574-4905461$ & $05: 15: 05.6$ & $-49: 05: 47$ & $g 4$ \\
\hline $2 M A S X J 05142945-4905534$ & $05: 14: 29.3$ & $-49: 05: 54$ & 93 \\
\hline
\end{tabular}


D. Bettoni et al.: Spectroscopic data for galaxies in eight nearby clusters, Online Material p 3

Table 4. Observed parameters of the standard and cluster galaxies.

\begin{tabular}{|c|c|c|c|c|c|c|c|c|c|c|c|c|c|c|c|c|}
\hline \multirow[t]{2}{*}{ Galaxy } & \multicolumn{10}{|c|}{ LRS data } & \multicolumn{6}{|c|}{ IRS data } \\
\hline & $c z$ & $Q$ & Refs. & $k_{B}$ & $k_{V}$ & $k_{r}$ & D4000 & $\mathrm{Mg}_{2}$ & $\mathrm{NaD}$ & Run & $c z$ & $\epsilon$ & $\sigma$ & $\epsilon$ & $\mathrm{Mg}_{2}$ & Comments \\
\hline \multicolumn{17}{|l|}{ Std Gal } \\
\hline N541 & 5427 & 1 & $a, b$ & .08 & .02 & -.03 & 1.76 & .296 & 3.4 & 5 & - & - & - & - & - & - \\
\hline N720 & 1758 & 1 & $a, b$ & .02 & .01 & .00 & - & .318 & 4.5 & 2 & 1729 & 17 & 223 & 74 & .323 & - \\
\hline N1395 & 1699 & 1 & $a, b$ & - & .01 & .00 & - & .316 & 4.9 & 1 & 1667 & 38 & 275 & 28 & .344 & - \\
\hline N1399 & 1450 & 1 & $\mathrm{a}, \mathrm{c}$ & - & .01 & .00 & - & .341 & 5.3 & 2 & 1445 & 51 & 364 & 74 & .336 & - \\
\hline N1403 & 4292 & 1 & $a, d$ & - & .02 & .00 & 1.82 & .281 & 3.2 & 2 & 4281 & 36 & 178 & 43 & .265 & - \\
\hline N1406 & 1357 & 1 & $\mathrm{e}$ & .02 & .01 & .00 & 2.17 & .266 & 2.8 & 2 & - & - & - & - & - & - \\
\hline N1426 & 1409 & 1 & $\mathrm{a}, \mathrm{f}$ & - & .01 & .00 & - & - & 3.9 & 2 & 1425 & 17 & 156 & 23 & .260 & $\Delta V=172$ \\
\hline N1726 & 3964 & 1 & $\mathrm{a}, \mathrm{g}$ & - & - & - & - & .288 & 4.1 & 5 & - & - & - & - & - & - \\
\hline $\mathrm{N} 2340$ & 5971 & 1 & b & - & - & - & 2.48 & .335 & 5.8 & 3 & 5938 & 37 & 241 & 33 & .337 & - \\
\hline N2974 & - & - & - & - & - & - & - & - & - & 4 & 1980 & 51 & 253 & 35 & .289 & - \\
\hline N2986 & 2336 & 1 & $\mathrm{a}, \mathrm{b}$ & - & .01 & .00 & - & .319 & 5.3 & 1 & - & - & - & - & - & - \\
\hline E462G15 & 5751 & 1 & $\mathrm{a}, \mathrm{h}$ & - & .04 & .01 & 1.87 & .292 & 3.9 & 2 & 5812 & 75 & 263 & 15 & .272 & - \\
\hline N7507 & 1538 & 1 & $\mathrm{~d}$ & - & .01 & .01 & - & .335 & 5.0 & 2 & 1578 & 18 & 206 & 26 & .325 & - \\
\hline N7562 & 3583 & 1 & b & - & .03 & .02 & 2.47 & .294 & 4.7 & 2 & 3606 & 58 & 242 & 17 & .288 & - \\
\hline N7619 & 3804 & 1 & $a, b$ & .05 & .03 & .02 & 1.82 & .320 & 4.9 & 2 & 3801 & 41 & 275 & 35 & .312 & - \\
\hline \multicolumn{17}{|l|}{ Abell 98} \\
\hline F1g20 & $32104^{a}$ & 4 & - & .57 & - & - & 1.92 & .207 & 1.7 & 4 & - & - & - & - & - & - \\
\hline F1g22 & 30852 & 3 & - & .60 & - & - & 2.24 & .187 & 3.0 & 4 & - & - & - & - & - & - \\
\hline F1g30 & 31358 & 2 & - & .56 & - & - & 1.82 & .185 & 3.1 & 4 & - & - & - & - & - & - \\
\hline F1g31 & 29984 & 2 & - & .60 & - & - & 2.23 & .243 & 2.1 & 4 & - & - & - & - & - & - \\
\hline F1g38 & $31021^{b}$ & 2 & - & .60 & - & - & 2.12 & .231 & 2.4 & 4 & - & - & - & - & - & - \\
\hline F1g43 & 33099 & 3 & - & .61 & - & - & 2.19 & .216 & 1.6 & 4 & - & - & - & - & - & - \\
\hline F1g44 & $30605^{c}$ & 2 & - & - & - & - & - & - & - & 4 & - & - & - & - & - & EL \\
\hline F1g58 & 30654 & 1 & - & - & - & - & - & - & - & 5 & - & - & - & - & - & EL \\
\hline F1g65 & 31049 & 2 & - & .62 & - & - & 2.08 & .258 & 2.5 & 4 & - & - & - & - & - & - \\
\hline F1g76 & 30898 & 2 & - & .57 & - & - & 1.93 & .283 & 4.9 & 5 & - & - & - & - & - & - \\
\hline F1g77 & $42272^{d}$ & 2 & - & .55 & - & - & 1.51 & .178 & 3.0 & 4 & - & - & - & - & - & - \\
\hline F1g80 & 32312 & 2 & - & .57 & - & - & 1.91 & .226 & 2.4 & 5 & - & - & - & - & - & - \\
\hline F1g88 & 32122 & 1 & - & .61 & - & - & 1.93 & .258 & 3.9 & 4,5 & - & - & - & - & - & - \\
\hline $\mathrm{F} 2 \mathrm{~g} 1$ & 30484 & 3 & - & .40 & - & - & 1.45 & .119 & 2.5 & 5 & - & - & - & - & - & $\mathrm{H} \beta$ abs. \\
\hline $\mathrm{F} 2 \mathrm{~g} 1 \mathrm{~A}$ & 31475 & 2 & - & .51 & - & - & 1.92 & .201 & 2.6 & 5 & - & - & - & - & - & - \\
\hline F2g3 & 31605 & 2 & - & .52 & - & - & 1.83 & .181 & 2.9 & 5 & - & - & - & - & - & - \\
\hline $\mathrm{F} 2 \mathrm{~g} 5$ & 31100 & 2 & - & .51 & - & - & 1.85 & .239 & 2.3 & 5 & - & - & - & - & - & - \\
\hline $\mathrm{F} 2 \mathrm{~g} 7 \mathrm{~A}$ & 31005 & 3 & - & .51 & - & - & 1.85 & .243 & 3.4 & 5 & - & - & - & - & - & - \\
\hline $\mathrm{F} 2 \mathrm{~g} 7 \mathrm{~B}$ & 30510 & 1 & - & .52 & - & - & 1.68 & .218 & 1.8 & 5 & - & - & - & - & - & - \\
\hline F2g9 & 30151 & 3 & - & .53 & - & - & 1.92 & .238 & 3.6 & 5 & - & - & - & - & - & - \\
\hline F3g1 & 17871 & 1 & - & .29 & - & - & 1.89 & .279 & 2.9 & 5 & - & - & - & - & - & - \\
\hline F3g2 & 30169 & 2 & - & .57 & - & - & 1.76 & .233 & 2.6 & 5 & - & - & - & - & - & - \\
\hline F3g3 & $31164^{e}$ & 2 & - & .48 & - & - & 1.76 & .217 & 3.3 & 5 & - & - & - & - & - & - \\
\hline F3g4 & 10988 & 1 & - & - & - & - & - & - & - & 5 & - & - & - & - & - & EL \\
\hline F3g5 & 30343 & 2 & - & .41 & - & - & 1.35 & .157 & 2.3 & 5 & - & - & - & - & - & - \\
\hline F3g7 & 31284 & 2 & - & .49 & - & - & 1.85 & .261 & 2.0 & 5 & - & - & - & - & - & - \\
\hline F10g5 & 35868 & 1 & - & .55 & - & - & 1.82 & .205 & 1.7 & 5 & - & - & - & - & - & - \\
\hline F10g6 & 31037 & 1 & - & .52 & - & - & 1.87 & .211 & 2.4 & 5 & - & - & - & - & - & - \\
\hline F10g7 & 30370 & 1 & - & .49 & - & - & 1.68 & .174 & 3.6 & 5 & - & - & - & - & - & - \\
\hline F10g8 & 32834 & 1 & - & .52 & - & - & 1.76 & .201 & 2.4 & 5 & - & - & - & - & - & - \\
\hline F10g9 & 31094 & 1 & - & .45 & - & - & 1.53 & .161 & 2.1 & 5 & - & - & - & - & - & - \\
\hline \multicolumn{17}{|l|}{ Abell 119} \\
\hline D26 & 13539 & 1 & - & - & .08 & .03 & 1.87 & .259 & 3.8 & 2 & 13462 & 24 & 173 & 66 & .246 & - \\
\hline D36 & 13958 & 2 & - & .26 & .04 & .03 & 1.93 & .269 & 2.7 & 4 & - & - & - & - & - & - \\
\hline D37 & 12959 & 1 & $1,3,4$ & - & .08 & .03 & - & .319 & 4.8 & 1 & 12872 & 26 & 240 & 30 & .309 & $\Delta V=55$ \\
\hline D38 & 12591 & 1 & 1 & .22 & .04 & - & 1.88 & .269 & 2.5 & 4 & - & - & - & - & - & - \\
\hline D41 & 12408 & 1 & 1,3 & - & .07 & .03 & - & - & 3.9 & 1 & 12342 & 38 & 173 & 22 & .316 & $\Delta V=250$ \\
\hline $\mathrm{D} 44^{f}$ & 13184 & 1 & 1 & - & .06 & .02 & - & .298 & 3.0 & 1 & 13081 & 66 & 201 & 35 & .315 & EL \\
\hline D45 & 12660 & 1 & - & - & - & - & - & - & - & 1 & - & - & - & - & - & EL \\
\hline D47 & 14521 & 3 & 1 & .22 & .10 & .04 & 1.27 & .281 & 2.8 & 2 & 14628 & 40 & 194 & 37 & .261 & $\Delta V=186$ \\
\hline D49 & 13810 & 2 & 1 & - & .09 & .05 & 1.62 & .219 & 2.5 & 2 & 13733 & 67 & 291 & 43 & .233 & - \\
\hline D51 & 12540 & 1 & 1 & .24 & .03 & - & 1.96 & .273 & 4.2 & 4 & - & - & - & - & - & - \\
\hline D52 & 13413 & 1 & $1,2,3,5$ & - & .08 & .03 & 1.81 & - & 4.4 & 1,2 & 13447 & 50 & 264 & 30 & .331 & - \\
\hline
\end{tabular}


D. Bettoni et al.: Spectroscopic data for galaxies in eight nearby clusters, Online Material p 4

Table 4. continued.

\begin{tabular}{|c|c|c|c|c|c|c|c|c|c|c|c|c|c|c|c|c|}
\hline \multirow[t]{2}{*}{ Galaxy } & \multicolumn{10}{|c|}{ LRS data } & \multicolumn{6}{|c|}{ IRS data } \\
\hline & $c z$ & Q & Refs. & $k_{B}$ & $k_{V}$ & $k_{r}$ & D4000 & $\mathrm{Mg}_{2}$ & $\mathrm{NaD}$ & Run & $c z$ & $\epsilon$ & $\sigma$ & $\epsilon$ & $\mathrm{Mg}_{2}$ & Comments \\
\hline D60 & 11475 & 2 & $1,2,3,6$ & - & .06 & .02 & - & .288 & 4.1 & 1 & 11565 & 56 & 331 & 25 & .302 & - \\
\hline D62 & 12990 & 3 & 1 & - & .07 & .03 & - & .313 & 3.4 & 1 & 13077 & 28 & 161 & 19 & .321 & $\Delta V=70$ \\
\hline D66 & 13318 & 1 & $1,2,3,6$ & - & .07 & .02 & - & .295 & 4.8 & 1 & 13356 & 50 & 270 & 55 & .317 & - \\
\hline D68 & 12143 & 2 & 1 & - & .07 & .02 & - & .264 & 4.4 & 1 & 12225 & 44 & 220 & 42 & .308 & $\Delta V=380$ \\
\hline $\mathrm{D} 74^{g}$ & 12663 & 1 & 1 & - & .05 & .01 & - & .252 & 3.5 & 1 & 12647 & 61 & 249 & 28 & .240 & - \\
\hline D75 ${ }^{h}$ & 11532 & 1 & 1,5 & - & .05 & .01 & - & .261 & 2.7 & 1,2 & 11560 & 66 & 167 & 31 & .256 & - \\
\hline D93 ${ }^{i}$ & 11703 & 2 & 1,5 & - & .05 & .02 & - & .297 & 4.5 & 1 & 11705 & 48 & 210 & 72 & .272 & - \\
\hline D94 & 12553 & 1 & 1,5 & .23 & .04 & - & 2.03 & .264 & 3.0 & 4 & - & - & - & - & - & - \\
\hline $\mathrm{D} 99^{j}$ & 13698 & 2 & 1 & - & .07 & .05 & - & - & 2.8 & 1,2 & 13540 & 59 & 268 & 34 & .288 & - \\
\hline D102 & 13230 & 1 & 1 & - & .07 & .04 & 2.00 & .161 & 4.8 & 1,2 & 13258 & 61 & 196 & 48 & .178 & - \\
\hline D105 & 13335 & 1 & $1,2,3$ & - & .08 & .03 & 1.67 & - & 3.7 & 1 & 13350 & 62 & 313 & 89 & .286 & - \\
\hline $\mathrm{D} 107^{k}$ & 13184 & 2 & 1 & .16 & .06 & .01 & 1.00 & - & 3.4 & 2 & 13085 & 68 & 157 & 35 & .316 & $\Delta V=107$ \\
\hline D109 ${ }^{l}$ & 13029 & 1 & 1,5 & - & .05 & .01 & - & .202 & 3.3 & 1,2 & 13021 & 57 & 224 & 8 & .195 & - \\
\hline D111 & 12615 & 1 & - & .20 & .08 & .04 & 1.55 & .262 & 4.2 & 2 & 12585 & 70 & 249 & 54 & .255 & - \\
\hline D112 $2^{m}$ & 14560 & 3 & 1 & .19 & .06 & 0.02 & 1.43 & .196 & 2.6 & 2 & 14650 & 72 & 219 & 45 & .227 & - \\
\hline $\mathrm{D} 114^{n}$ & 13312 & 2 & 1 & - & .07 & .03 & 2.00 & .288 & 3.9 & 2 & 13375 & 40 & 128 & 11 & - & $\Delta V=90$ \\
\hline \multicolumn{17}{|c|}{ Abell 3125} \\
\hline D9 & 18508 & 1 & - & - & .14 & .08 & 1.45 & 261 & - & 1 & - & - & - & - & - & - \\
\hline D11 & $8682^{\circ}$ & 1 & 1,8 & - & .02 & .03 & - & - & - & 1 & - & - & - & - & - & EL \\
\hline D14 & 15532 & 1 & - & - & .15 & .12 & - & - & - & 1 & - & - & - & - & - & EL \\
\hline D46 & 17900 & 3 & 9 & - & .13 & .08 & 1.64 & .270 & 4.4 & 1 & 17830 & 38 & 253 & 24 & .276 & $\Delta V=50$ \\
\hline D47 & 17816 & 2 & 9 & - & .13 & .09 & 1.76 & .241 & 3.8 & 1 & 17745 & 31 & 299 & 42 & .269 & - \\
\hline D48 & 17346 & 1 & 9 & - & .12 & .08 & 2.14 & .189 & 3.0 & 1 & - & - & - & - & - & - \\
\hline D51 & 18790 & 1 & - & - & .14 & .09 & 2.08 & .282 & 4.3 & 1,2 & 18845 & 36 & 213 & 46 & .300 & - \\
\hline D60 & 17587 & 1 & 9 & - & .13 & .09 & 1.54 & .255 & 3.6 & 1 & 17478 & 45 & 291 & 59 & .281 & - \\
\hline D77 & 17440 & 2 & - & - & .11 & .07 & - & - & - & 1 & 17298 & 70 & 261 & 67 & - & EL \\
\hline D88 & 17675 & 2 & - & - & .14 & .09 & 1.06 & .332 & 4.7 & 1 & 17693 & 71 & 332 & 85 & .310 & $\Delta V=189$ \\
\hline D93 & 17485 & 1 & 9 & - & .11 & .07 & 1.22 & .259 & 2.9 & 2 & 17621 & 35 & 287 & 77 & .271 & - \\
\hline D95 & - & - & - & & - & - & - & - & - & - & 17866 & 61 & 335 & 34 & .263 & $\Delta V=73$ \\
\hline D96 & 17702 & 3 & 9 & - & .13 & .10 & 2.33 & . 198 & 2.0 & 1 & 17652 & 48 & 239 & 22 & .215 & - \\
\hline D103 & 17786 & 1 & - & - & .13 & - & 1.54 & . 177 & 3.0 & 2 & 17766 & 49 & 288 & 11 & .185 & - \\
\hline D104 & 18099 & 1 & - & - & .14 & .09 & 1.67 & 237 & 2.8 & 1 & - & - & - & - & - & - \\
\hline D129 & 18890 & 1 & 9 & - & .13 & .09 & 1.47 & .231 & 2.6 & 1 & - & - & - & - & - & - \\
\hline D130 & 19112 & 2 & 9 & - & .13 & .09 & 1.53 & .281 & 3.2 & 1 & 19049 & 27 & 236 & 25 & .271 & - \\
\hline D140 & 18917 & 2 & 9 & - & .15 & .10 & 1.64 & .211 & 3.9 & 1 & 18945 & 39 & 212 & 5 & .209 & - \\
\hline D160 & 18361 & 1 & 9 & - & .15 & .10 & 1.39 & 263 & 3.2 & 1 & 18459 & 46 & 262 & 61 & - & EL \\
\hline D161 & 18115 & 2 & - & - & .14 & .10 & 1.68 & .315 & 2.8 & 1,2 & 17964 & 79 & 328 & 35 & .336 & - \\
\hline \multicolumn{17}{|c|}{ Abell 3330} \\
\hline G1 & 27522 & 2 & - & - & .10 & - & 1.54 & .244 & 4.0 & 1 & - & - & - & - & - & - \\
\hline G2 & 26446 & 2 & - & - & .10 & - & 1.00 & .226 & 1.6 & 1 & - & - & - & - & - & - \\
\hline G3 & 27358 & 2 & - & - & .15 & - & 2.66 & .260 & 3.2 & 1 & - & - & - & - & - & - \\
\hline G4 & 26586 & 3 & - & - & .13 & - & 1.97 & .242 & - & 1 & - & - & - & - & - & - \\
\hline G5 & 28262 & 1 & - & - & .17 & - & 1.45 & .314 & 5.5 & 1 & - & - & - & - & - & - \\
\hline G6 & 27140 & 1 & - & - & .14 & - & 1.68 & .258 & 3.8 & 1 & - & - & - & - & - & - \\
\hline G7 & 26222 & 1 & - & - & .11 & - & - & - & - & 1 & - & - & - & - & - & EL \\
\hline G21 & 26463 & 1 & - & - & .19 & - & 1.50 & .324 & 2.9 & 1 & - & - & - & - & - & - \\
\hline G22 & 28251 & 1 & - & - & .20 & - & 1.44 & .247 & 3.0 & 1 & - & - & - & - & - & - \\
\hline \multicolumn{17}{|c|}{ Abell 1069} \\
\hline D3 & 19336 & 1 & - & - & .10 & .07 & 1.16 & .232 & 4.8 & 1 & - & - & - & - & - & - \\
\hline D9 & 19478 & 3 & - & - & .11 & .07 & 2.44 & .234 & 3.6 & 1 & - & - & - & - & - & - \\
\hline D12 & 16624 & 2 & 7 & - & .08 & .07 & 1.66 & .253 & 2.3 & 1 & - & - & - & - & - & - \\
\hline D14 & 16656 & 1 & - & - & .08 & .07 & 1.38 & .275 & 3.6 & 1 & - & - & - & - & - & - \\
\hline D15 & 19737 & 1 & 1,7 & - & .10 & .07 & 1.51 & .235 & 3.9 & 1,6 & 19709 & 23 & 239 & 9 & .292 & - \\
\hline D16 & 20110 & 4 & 1 & - & .09 & .07 & 1.00 & . 140 & 5.2 & 1 & - & - & - & - & - & - \\
\hline D19 & 18030 & 1 & 1,7 & - & .10 & .08 & 1.10 & .224 & 3.7 & 1,6 & 18040 & 31 & 220 & 11 & .261 & - \\
\hline D21 & 19817 & 2 & 1,7 & - & .11 & .06 & 1.20 & .319 & 3.2 & 1,6 & 19345 & 31 & 357 & 9 & .328 & - \\
\hline D25 & 20331 & 1 & 1,7 & - & .09 & .04 & 1.82 & .212 & 2.3 & 1,6 & 20287 & 19 & 275 & 7 & .219 & - \\
\hline D29 & 20051 & 1 & 1,7 & - & .10 & .07 & 2.00 & .259 & 4.5 & 1,6 & 20067 & 17 & 236 & 7 & .283 & - \\
\hline D30 & 20430 & 1 & 1 & - & .08 & .06 & 1.22 & .219 & 3.4 & 1 & - & - & - & - & - & - \\
\hline D38 & 19329 & 2 & - & - & .11 & .08 & - & .244 & - & 1,6 & 19432 & 19 & 295 & 7 & - & - \\
\hline
\end{tabular}


D. Bettoni et al.: Spectroscopic data for galaxies in eight nearby clusters, Online Material p 5

Table 4. continued.

\begin{tabular}{|c|c|c|c|c|c|c|c|c|c|c|c|c|c|c|c|c|}
\hline \multirow[t]{2}{*}{ Galaxy } & \multicolumn{10}{|c|}{ LRS data } & \multicolumn{6}{|c|}{ IRS data } \\
\hline & $c z$ & $Q$ & Refs. & $k_{B}$ & $k_{V}$ & $k_{r}$ & D4000 & $\mathrm{Mg}_{2}$ & $\mathrm{NaD}$ & Run & $c z$ & $\epsilon$ & $\sigma$ & $\epsilon$ & $\mathrm{Mg}_{2}$ & Comments \\
\hline D39 & 19729 & 3 & - & - & .09 & .07 & - & .192 & 1.7 & 1 & - & - & - & - & - & - \\
\hline D41 & 11644 & 1 & - & - & - & - & - & - & - & 1 & - & - & - & - & - & EL \\
\hline D46 & 19700 & 2 & - & - & .09 & .05 & 1.97 & .248 & 4.8 & 1,6 & 19653 & 19 & 231 & 8 & .307 & - \\
\hline D47 & - & - & - & - & - & - & - & - & - & 6 & 18931 & 14 & 149 & 10 & .250 & - \\
\hline \multicolumn{17}{|c|}{ Abell 1983} \\
\hline D6 & 17547 & 1 & 10 & - & - & - & 1.66 & .186 & 2.0 & 3 & - & - & - & - & - & - \\
\hline D10 & 13156 & 1 & 10 & - & .07 & - & 1.60 & .303 & 5.3 & 3 & - & - & - & - & - & - \\
\hline D15 & 13589 & 1 & 10 & - & .07 & - & 2.11 & .292 & 4.3 & 3,6 & 13676 & 20 & 323 & 7 & .323 & - \\
\hline D23 & 12782 & 1 & 10 & - & .05 & - & 1.46 & .154 & 2.3 & 3 & - & - & - & - & - & - \\
\hline $\mathrm{D} 24$ & 13504 & 1 & 10 & - & .07 & - & 1.59 & .241 & 4.4 & 3 & - & - & - & - & - & - \\
\hline D26 & 17822 & 1 & 10 & - & - & - & 1.90 & .294 & 4.4 & 3 & - & - & - & - & - & - \\
\hline D28 & 6251 & 1 & 10 & - & .04 & - & 1.59 & .180 & 2.0 & 3 & - & - & - & - & - & - \\
\hline D35 & 13659 & 1 & 10 & - & .08 & - & 2.36 & .237 & 4.1 & 3 & - & - & - & - & - & - \\
\hline D46 & 13532 & 1 & 10 & - & .07 & - & 2.15 & .280 & 2.7 & 3 & - & - & - & - & - & - \\
\hline D54 & 13167 & 2 & 7 & - & .07 & - & 1.79 & .297 & 4.7 & 3,6 & 12979 & 14 & 232 & 7 & .298 & - \\
\hline D56 & 13481 & 1 & 10 & - & .07 & - & 2.04 & .285 & 4.8 & 3,6 & 13583 & 20 & 198 & 9 & .265 & - \\
\hline D58 & 13606 & 1 & 11 & - & .07 & - & 2.03 & .212 & 2.6 & 3 & - & - & - & - & - & - \\
\hline D77 & - & - & - & - & - & - & - & - & - & 6 & 13844 & 15 & 215 & 8 & .266 & - \\
\hline D78 & 13781 & 1 & 7 & - & .06 & - & 1.85 & .306 & 3.9 & 3,6 & 13639 & 15 & 212 & 8 & .277 & - \\
\hline D84 & 13894 & 1 & 11 & - & .08 & - & 1.96 & .281 & 4.8 & 3 & - & - & - & - & - & - \\
\hline D105 & 13310 & 1 & 10 & - & .06 & - & 1.98 & .264 & 4.3 & 3,6 & 13387 & 20 & 323 & 7 & .323 & - \\
\hline \multicolumn{17}{|c|}{ Abell 2151} \\
\hline D4 & 9986 & 3 & 10 & .18 & .06 & - & 1.91 & .219 & 1.9 & 4 & - & - & - & - & - & - \\
\hline D7 & 10055 & 1 & 2 & .20 & .07 & - & 2.23 & .290 & 3.2 & 4 & - & - & - & - & - & - \\
\hline D9 & 10248 & 1 & 10 & .18 & .06 & - & 1.90 & .261 & 1.7 & 4 & - & - & - & - & - & - \\
\hline D15 & 9966 & 1 & 10 & .19 & .06 & - & 1.96 & .281 & 2.6 & 4 & - & - & - & - & - & - \\
\hline D40 & 10330 & 1 & 12 & .19 & .04 & - & 2.12 & .268 & 3.1 & 4,6 & 10050 & 15 & 230 & 7 & .271 & - \\
\hline D62 & 9338 & 1 & 2 & .18 & .06 & - & 2.36 & .233 & 3.2 & 4 & - & - & - & - & - & - \\
\hline D63 & 10830 & 1 & 10 & .21 & .07 & - & 2.43 & .281 & 3.1 & 4,6 & 10455 & 16 & 195 & 9 & .262 & - \\
\hline D64 & 10432 & 2 & 10 & .21 & .07 & - & 1.97 & - & 5.0 & 4 & - & - & - & - & - & - \\
\hline D65 & - & - & - & - & - & - & - & - & - & 6 & 10426 & 13 & 291 & 6 & .305 & - \\
\hline D66 & - & - & - & - & - & - & - & - & - & 6 & 9643 & 9 & 186 & 6 & .270 & - \\
\hline D78 & 10262 & 1 & - & .13 & .05 & - & - & - & - & 4 & - & - & - & - & - & EL \\
\hline D134 & - & - & - & - & - & - & - & - & - & 6 & 11074 & 50 & 318 & 11 & .302 & - \\
\hline \multicolumn{17}{|c|}{ DC 2103-39 } \\
\hline D3 & 16114 & 1 & - & .26 & .13 & .03 & 1.33 & .268 & 4.2 & 2 & 16152 & 35 & 185 & 27 & .272 & $\Delta V=150$ \\
\hline D14 & 9374 & 1 & - & .13 & .05 & .02 & 1.28 & .329 & 4.9 & 2 & 9343 & 40 & 332 & 58 & .327 & - \\
\hline D15 & 15034 & 1 & - & .23 & .09 & .04 & 1.32 & .335 & 3.2 & 2 & 15038 & 39 & 237 & 30 & .316 & $\Delta V=103$ \\
\hline D18 & 9319 & 2 & - & - & .05 & .03 & - & - & - & 2 & 9208 & 30 & 137 & 38 & - & $\mathrm{EL}, \Delta V=122$ \\
\hline D20 & 9287 & 1 & - & .11 & .04 & .02 & 1.00 & .185 & 1.8 & 2 & 9353 & 19 & 154 & 38 & .191 & - \\
\hline D21 & 9161 & 1 & - & .15 & .06 & .03 & 1.49 & .229 & 2.5 & 2 & 9400 & 31 & 242 & 64 & .218 & $\Delta V=91$ \\
\hline D23 & 9279 & 1 & - & .13 & .07 & .03 & 1.25 & .273 & 2.6 & 2 & 9245 & 62 & 260 & 52 & .244 & $\Delta V=270$ \\
\hline D38 & 15820 & 1 & - & .22 & .08 & .04 & 1.60 & .232 & 3.0 & 2 & 15742 & 52 & 275 & 47 & .212 & - \\
\hline D39 & 15869 & 3 & - & .26 & .09 & .04 & 1.19 & .252 & 1.0 & 2 & 15892 & 51 & 313 & 23 & .268 & - \\
\hline D40 & 15060 & 1 & - & .22 & .08 & .04 & 1.47 & .249 & 3.9 & 2 & 14992 & 33 & 223 & 17 & .260 & - \\
\hline D42 & 14719 & 1 & - & .20 & .10 & - & 1.47 & .240 & 3.6 & 2 & 14700 & 24 & 206 & 71 & .249 & - \\
\hline
\end{tabular}


D. Bettoni et al.: Spectroscopic data for galaxies in eight nearby clusters, Online Material p 6

Table 4. continued.

\begin{tabular}{|c|c|c|c|c|c|c|c|c|c|c|c|c|c|c|c|c|}
\hline \multirow{2}{*}{ Galaxy } & \multicolumn{9}{|c|}{ LRS data } & \multicolumn{7}{|c|}{ IRS data } \\
\hline & $c z$ & $Q$ & Refs. & $k_{B}$ & $k_{V}$ & $k_{r}$ & D4000 & $\mathrm{Mg}_{2}$ & $\mathrm{NaD}$ & Run & $c z$ & $\epsilon$ & $\sigma$ & $\epsilon$ & $\mathrm{Mg}_{2}$ & Comments \\
\hline $\bar{D} 53$ & 26506 & $\frac{2}{1}$ & - & - & .13 & - & 1.97 & .287 & 3.7 & 2 & 26558 & 60 & 316 & 23 & - & - \\
\hline D60 & 15635 & 2 & - & .22 & .11 & .05 & 1.25 & .224 & 3.7 & 2 & 15626 & 32 & 177 & 58 & .232 & - \\
\hline D61 & 15369 & 2 & - & .20 & .10 & .04 & 1.33 & .278 & 3.1 & 2 & 15326 & 33 & 200 & 69 & - & - \\
\hline D62 & 15149 & 1 & - & - & .07 & .03 & 1.07 & .227 & 1.4 & 2 & 15079 & 23 & 176 & 17 & .230 & - \\
\hline D63 & 15003 & 2 & - & - & .08 & .03 & 1.16 & - & 1.9 & 2 & 14937 & 23 & 147 & 24 & .271 & - \\
\hline D66 & 9527 & 2 & - & - & .04 & .02 & 1.18 & .166 & 0.8 & 2 & 9581 & 39 & 235 & 23 & .177 & - \\
\hline D71 & 14836 & 1 & - & - & .08 & .04 & 1.37 & .249 & 4.1 & 2 & 14832 & 44 & 207 & 33 & .258 & - \\
\hline D73 & - & - & - & - & - & - & - & - & - & - & 14940 & 38 & 260 & 17 & .276 & - \\
\hline D76 & 15230 & 3 & - & .20 & .08 & .03 & 1.66 & .187 & 4.0 & 2 & - & - & - & - & - & - \\
\hline D102 & - & - & - & - & - & - & - & - & - & - & 15324 & 39 & 207 & 55 & - & $\Delta V=92$ \\
\hline
\end{tabular}

References for the redshift. (A) Standard Galaxies: ${ }^{a}$ JFK; ${ }^{b}$ Smith et al. (2000); ${ }^{c}$ Graham et al. (1998); ${ }^{d}$ de Vaucouleurs et al. (1991, RC3);

${ }^{e}$ Bureau et al. (1996); ${ }^{f}$ Lauberts \& Valentijn (1989, ESO-Uppsala Catalog); ${ }^{g}$ Simien and Prugniel (1997); ${ }^{h}$ da Costa et al. (1991). (B) Cluster Galaxies. 1: Katgert et al. (1998, ENACS Catalog); 2, de Vaucouleurs et al. (1991, RC3); 3, Huchra et al. (1999, CfA Catalog); 4, Zabludoff et al. (1993); 5, Paturel et al. (2003, LEDA Catalogue); 6, Postman \& Lauer (1995); 7, Beers et al. (1991); 8, Lauberts \& Valentijn (1989, ESO-Uppsala Catalog); 9, Caldwell \& Rose (1997); 10, Dressler \& Shectman (1988); 11, Zabludoff et al. (1990); 12, Davoust \& Considere (1995).

Notes on discrepant redshift results:

- ${ }^{a}$ In agreement with B82, but $394 \mathrm{~km} \mathrm{~s}^{-1}$ higher than in Zabludoff et al. (1990).

$-{ }^{b} 634 \mathrm{~km} \mathrm{~s}^{-1}$ higher than in Z90.

- ${ }^{c}$ Our value, determined form the emission lines, agrees with Z90, but it is $735 \mathrm{~km} \mathrm{~s}^{-1}$ lower than in Beers et al. (1982).

- ${ }^{d}$ Our value agrees with Z90, and is $9826 \mathrm{~km} \mathrm{~s}^{-1}$ higher than in Beers et al. (1982).

- ${ }^{e}$ The redshift we find for this galaxy is $368 \mathrm{~km} \mathrm{~s}^{-1}$ higher than in Beers et al. (1982), but grossly discrepant with Zabludoff et al.(1990).

- ${ }^{f}$ Observed in LRS and IRS modes with concordant results. Our LRS value is $337 \mathrm{~km} \mathrm{~s}^{-1}$ higher than in the ENACS Catalogue.

- ${ }^{g}$ Observed in LRS and IRS modes with concordant results. Our LRS value is $1038 \mathrm{~km} \mathrm{~s}^{-1}$ lower than in the ENACS Catalogue, and very close to that reported by Fouqué et al. (1992).

- ${ }^{h}$ Observed twice in LRS mode and in IRS mode, with concordant results. Our LRS value is $1330 \mathrm{~km} \mathrm{~s}^{-1}$ lower than in the ENACS Catalogue.

$-{ }^{i}$ Observed in LRS and IRS modes with concordant results. Our LRS value is $1357 \mathrm{~km} \mathrm{~s}^{-1}$ lower than in the ENACS Catalogue. The redshift reported by Falco et al. (1999) is very close to our value, whereas that reported by Fouqué et al. (1992) does not agree with any of previous determinations.

- ${ }^{j}$ Observed in LRS and IRS modes with concordant results. Our LRS value is $1226 \mathrm{~km} \mathrm{~s}^{-1}$ higher than in the ENACS Catalogue.

- ${ }^{k}$ Observed in LRS and IRS modes with concordant results. Our LRS value is $1210 \mathrm{~km} \mathrm{~s}^{-1}$ lower than in the ENACS Catalogue. The redshift reported by Fouqué et al. (1992) is very close to our value.

- ${ }^{l}$ Observed in LRS and IRS modes with concordant results. Our LRS value is $2086 \mathrm{~km} \mathrm{~s}^{-1}$ higher than in the ENACS Catalogue.

- ${ }^{m}$ Observed in LRS and IRS modes with concordant results. Our LRS value is $2819 \mathrm{~km} \mathrm{~s}^{-1}$ higher than in the ENACS Catalogue.

- ${ }^{n}$ Observed in LRS and IRS modes with concordant results. Our LRS value is $5393 \mathrm{~km} \mathrm{~s}^{-1}$ lower than in the ENACS Catalogue.

- ${ }^{\circ}$ Our redshift is $9478 \mathrm{~km} \mathrm{~s}^{-1}$ lower than in the ENACS Catalogue, that is in agreement with the redshift given in the ESO-Uppsala catalog. Our value is from the detected emission lines. 
D. Bettoni et al.: Spectroscopic data for galaxies in eight nearby clusters, Online Material $p 7$

Table 6. The adopted, fully corrected, redshift and spectral indicators.

\begin{tabular}{|c|c|c|c|c|c|}
\hline Galaxy & $c z$ & D4000 & $\mathrm{NaD}$ & $\mathrm{Mg}_{2}$ & $\log \sigma$ \\
\hline \multicolumn{6}{|l|}{ Abell 98} \\
\hline F1g20 & 32104 & 2.02 & 3.2 & .270 & - \\
\hline F1g22 & 30852 & 2.34 & 4.5 & .250 & - \\
\hline F1g30 & 31358 & 1.92 & 4.6 & .248 & - \\
\hline F1g31 & 29984 & 2.33 & 3.6 & .306 & - \\
\hline F1g38 & 31021 & 2.22 & 3.9 & .294 & - \\
\hline F1g43 & 33099 & 2.29 & 3.1 & .279 & - \\
\hline F1g44 & 30605 & - & - & - & - \\
\hline F1g58 & 30654 & - & - & - & - \\
\hline F1g65 & 31049 & 2.18 & 4.0 & .321 & - \\
\hline F1g76 & 30898 & 2.03 & 6.4 & .346 & - \\
\hline F1g77 & 42272 & 1.66 & 4.6 & .242 & - \\
\hline F1g80 & 32312 & 2.01 & 3.9 & .289 & - \\
\hline F1g88 & 32122 & 2.03 & 5.4 & .321 & - \\
\hline F2g1 & 30484 & 1.55 & 4.0 & .182 & - \\
\hline $\mathrm{F} 2 \mathrm{~g} 1 \mathrm{~A}$ & 31475 & 2.02 & 4.1 & .264 & - \\
\hline F2g3 & 31605 & 1.93 & 4.4 & .244 & - \\
\hline F2g5 & 31100 & 1.95 & 3.8 & .302 & - \\
\hline $\mathrm{F} 2 \mathrm{~g} 7 \mathrm{~A}$ & 31005 & 1.95 & 4.9 & .306 & - \\
\hline $\mathrm{F} 2 \mathrm{~g} 7 \mathrm{~B}$ & 30510 & 1.78 & 3.3 & .281 & - \\
\hline F2g9 & 30151 & 2.02 & 5.1 & .301 & - \\
\hline F3g1 & 17871 & 1.93 & 4.1 & .332 & - \\
\hline F3g2 & 30169 & 1.86 & 4.1 & .296 & - \\
\hline F3g3 & 31164 & 1.86 & 4.8 & .280 & - \\
\hline F3g4 & 10988 & - & - & - & - \\
\hline F3g5 & 30343 & 1.45 & 3.8 & .220 & - \\
\hline F3g7 & 31284 & 1.95 & 3.5 & .324 & - \\
\hline F10g5 & 35868 & 1.92 & 3.2 & .268 & - \\
\hline F10g6 & 31037 & 1.97 & 3.9 & .274 & - \\
\hline F10g7 & 30370 & 1.78 & 5.1 & .237 & - \\
\hline F10g8 & 32834 & 1.86 & 3.9 & .264 & - \\
\hline F10g9 & 31094 & 1.63 & 3.6 & .224 & - \\
\hline \multicolumn{6}{|c|}{ Abell 119} \\
\hline D26 & 13462 & 1.90 & 4.0 & .263 & 2.238 \\
\hline D36 & 13958 & 1.96 & 3.8 & .317 & - \\
\hline D37 & 12872 & - & 5.0 & .326 & 2.380 \\
\hline D38 & 12591 & 1.91 & 3.6 & .317 & - \\
\hline D41 & 12342 & - & 4.1 & .333 & 2.238 \\
\hline D44 & 13081 & - & 3.2 & .332 & 2.303 \\
\hline D45 & 12660 & - & - & - & - \\
\hline D47 & 14628 & 1.30 & 3.0 & .278 & 2.288 \\
\hline D49 & 13733 & 1.65 & 2.7 & .250 & 2.464 \\
\hline D51 & 12540 & 1.99 & 5.3 & .321 & - \\
\hline D52 & 13447 & 1.84 & 4.6 & .348 & 2.422 \\
\hline D60 & 11565 & - & 4.3 & .319 & 2.520 \\
\hline D62 & 13077 & - & 3.6 & .338 & 2.207 \\
\hline D66 & 13356 & - & 5.0 & .334 & 2.431 \\
\hline D68 & 12225 & - & 4.6 & .325 & 2.342 \\
\hline D74 & 12647 & - & 3.7 & .257 & 2.396 \\
\hline D75 & 11560 & - & 2.9 & .273 & 2.223 \\
\hline D93 & 11705 & - & 4.7 & .289 & 2.322 \\
\hline D94 & 12553 & 2.06 & 4.1 & .312 & - \\
\hline D99 & 13540 & - & 3.0 & .305 & 2.428 \\
\hline D102 & 13258 & 2.03 & 5.0 & .195 & 2.292 \\
\hline D105 & 13350 & 1.70 & 3.9 & .303 & 2.496 \\
\hline D107 & 13085 & 1.03 & 3.6 & .333 & 2.196 \\
\hline
\end{tabular}

Table 6. continued.

\begin{tabular}{|c|c|c|c|c|c|}
\hline$\overline{\text { Galaxy }}$ & $c z$ & $\mathrm{D} 4000$ & $\mathrm{NaD}$ & $\overline{\mathrm{Mg}_{2}}$ & $\log \sigma$ \\
\hline D109 & 13021 & - & 3.5 & .212 & 2.350 \\
\hline D111 & 12585 & 1.58 & 4.4 & .272 & 2.396 \\
\hline D112 & 14650 & 1.46 & 2.8 & .244 & 2.340 \\
\hline D114 & 13375 & 2.03 & 4.1 & .336 & 2.107 \\
\hline \multicolumn{6}{|c|}{ Abel 3125} \\
\hline D9 & 18508 & 1.50 & - & .295 & - \\
\hline D11 & 8682 & - & - & - & - \\
\hline D14 & 15532 & - & - & - & - \\
\hline D46 & 17830 & 1.69 & 4.7 & .299 & 2.403 \\
\hline D47 & 17745 & 1.81 & 4.1 & .292 & 2.476 \\
\hline D48 & 17346 & 2.19 & 3.3 & .223 & - \\
\hline D51 & 18845 & 2.13 & 4.6 & .323 & 2.328 \\
\hline D60 & 17478 & 1.59 & 3.9 & .304 & 2.464 \\
\hline D77 & 17298 & - & - & - & 2.417 \\
\hline D88 & 17693 & 1.11 & 5.0 & .333 & 2.521 \\
\hline D93 & 17621 & 1.27 & 3.2 & .294 & 2.458 \\
\hline D95 & 17866 & - & - & .287 & 2.525 \\
\hline D96 & 17652 & 2.38 & 2.3 & .238 & 2.378 \\
\hline D103 & 17766 & 1.59 & 3.3 & .208 & 2.459 \\
\hline D104 & 18099 & 1.72 & 3.1 & .271 & - \\
\hline D129 & 18890 & 1.52 & 2.9 & .265 & - \\
\hline D130 & 19049 & 1.58 & 3.5 & .294 & 2.373 \\
\hline D140 & 18945 & 1.69 & 4.2 & .232 & 2.326 \\
\hline D160 & 18459 & 1.44 & 3.5 & .297 & 2.418 \\
\hline D161 & 17964 & 1.73 & 3.1 & .358 & 2.516 \\
\hline \multicolumn{6}{|c|}{ Abel 3330} \\
\hline g1 & 27522 & 1.63 & 4.5 & .285 & - \\
\hline g2 & 26446 & 1.09 & 2.1 & .267 & - \\
\hline g3 & 27358 & 2.75 & 3.7 & .301 & - \\
\hline g4 & 26586 & 2.06 & - & .283 & - \\
\hline g5 & 28262 & 1.54 & 6.0 & .355 & - \\
\hline g6 & 27140 & 1.77 & 4.3 & .299 & - \\
\hline g7 & 26222 & - & - & - & - \\
\hline g21 & 26463 & 1.59 & 3.4 & .365 & - \\
\hline g22 & 28251 & 1.53 & 3.5 & .288 & - \\
\hline \multicolumn{6}{|c|}{ Abell 1069} \\
\hline D3 & 19336 & 1.22 & 5.1 & .267 & - \\
\hline D9 & 19478 & 2.50 & 3.9 & .269 & - \\
\hline D12 & 16624 & 1.72 & 2.6 & .288 & - \\
\hline D14 & 16656 & 1.44 & 3.9 & .310 & - \\
\hline D15 & 19709 & 1.57 & 4.2 & .317 & 2.378 \\
\hline D16 & 20110 & 1.06 & 5.5 & .175 & - \\
\hline D19 & 18040 & 1.16 & 4.0 & .285 & 2.342 \\
\hline D21 & 19345 & 1.26 & 3.5 & .352 & 2.553 \\
\hline D25 & 20247 & 1.88 & 2.6 & .243 & 2,439 \\
\hline D29 & 20067 & 2.06 & 4.8 & .307 & 2.373 \\
\hline D30 & 20430 & 1.28 & 3.7 & .254 & - \\
\hline D38 & 19432 & - & - & .279 & 2.470 \\
\hline D39 & 19729 & - & 2.0 & .227 & - \\
\hline D41 & 11644 & - & - & - & - \\
\hline D46 & 19653 & 2.03 & 5.1 & .331 & 2.364 \\
\hline D47 & 18931 & - & - & .274 & 2.173 \\
\hline \multicolumn{6}{|c|}{ Abell 1983} \\
\hline D6 & 17547 & 1.69 & 2.2 & .214 & - \\
\hline D10 & 13156 & 1.63 & 5.5 & .331 & - \\
\hline D15 & 13676 & 2.14 & 4.5 & .340 & 2.509 \\
\hline
\end{tabular}


D. Bettoni et al.: Spectroscopic data for galaxies in eight nearby clusters, Online Material $p 8$

Table 6. continued.

\begin{tabular}{|c|c|c|c|c|c|}
\hline Galaxy & $c z$ & $\overline{\mathrm{D} 4000}$ & $\mathrm{NaD}$ & $\mathrm{Mg}_{2}$ & $\log \sigma$ \\
\hline D23 & 12782 & 1.49 & 2.5 & .182 & - \\
\hline D24 & 13504 & 1.62 & 4.6 & .269 & _ \\
\hline D26 & 17822 & 1.93 & 4.6 & .322 & - \\
\hline D28 & 6251 & 1.60 & 2.1 & .205 & _ \\
\hline D35 & 13659 & 2.39 & 4.3 & .265 & _ \\
\hline D46 & 13532 & 2.18 & 2.9 & .308 & - \\
\hline D54 & 12979 & 1.82 & 4.9 & .315 & 2.365 \\
\hline D56 & 13583 & 2.07 & 5.0 & .282 & 2.297 \\
\hline D58 & 13606 & 2.06 & 2.8 & .240 & - \\
\hline D77 & 13844 & - & - & .283 & 2.332 \\
\hline D78 & 13639 & 1.88 & 4.1 & .294 & 2.326 \\
\hline D84 & 13894 & 1.99 & 5.0 & .309 & - \\
\hline D105 & 13387 & 2.01 & 4.5 & .340 & 2.509 \\
\hline \multicolumn{6}{|c|}{ Abel 2151} \\
\hline D4 & 9986 & 1.92 & 2.9 & .264 & - \\
\hline D7 & 10055 & 2.24 & 4.2 & .335 & - \\
\hline D9 & 10248 & 1.91 & 2.7 & .306 & - \\
\hline D15 & 9966 & 1.97 & 3.6 & .326 & - \\
\hline D40 & 10050 & 2.13 & 4.1 & .285 & 2.362 \\
\hline D62 & 9338 & 2.37 & 4.2 & .278 & - \\
\hline D63 & 10455 & 2.44 & 4.1 & .276 & 2.290 \\
\hline D64 & 10432 & 1.98 & 6.0 & - & - \\
\hline D65 & 10426 & - & - & .319 & 2.464 \\
\hline D66 & 9643 & - & - & .284 & 2.270 \\
\hline D78 & 10262 & - & - & - & - \\
\hline D134 & 11074 & - & - & .316 & 2.502 \\
\hline \multicolumn{6}{|c|}{ DC2103-39 } \\
\hline D3 & 16152 & 1.37 & 4.5 & .294 & 2.267 \\
\hline D14 & 9343 & 1.31 & 5.0 & .342 & 2.521 \\
\hline D15 & 15038 & 1.36 & 3.5 & .338 & 2.375 \\
\hline D18 & 9208 & - & - & - & 2.137 \\
\hline D20 & 9353 & 1.01 & 1.9 & .206 & 2.188 \\
\hline D21 & 9400 & 1.50 & 2.6 & .233 & 2.384 \\
\hline D23 & 9245 & 1.26 & 2.7 & .259 & 2.415 \\
\hline D38 & 15742 & 1.64 & 3.3 & .234 & 2.439 \\
\hline D39 & 15892 & 1.23 & 1.3 & .290 & 2.496 \\
\hline D40 & 14992 & 1.51 & 4.2 & .282 & 2.348 \\
\hline D42 & 14700 & 1.51 & 3.9 & .271 & 2.314 \\
\hline D53 & 26558 & 2.06 & 4.3 & .348 & 2.500 \\
\hline D60 & 15626 & 1.29 & 4.0 & .254 & 2.248 \\
\hline D61 & 15326 & 1.37 & 3.4 & .311 & 2.301 \\
\hline D62 & 15079 & 1.11 & 1.7 & .252 & 2.246 \\
\hline D63 & 14937 & 1.20 & 2.2 & .293 & 2.167 \\
\hline D66 & 9581 & 1.19 & - & 192 & 2.371 \\
\hline D71 & 14832 & 1.41 & 4.4 & .280 & 2.316 \\
\hline D73 & 14940 & - & - & .298 & 2.415 \\
\hline D76 & 15230 & 1.70 & 4.3 & . 220 & - \\
\hline D102 & 15324 & - & - & - & 2.316 \\
\hline
\end{tabular}

